\title{
NGC 6752 AGB stars revisited
}

\section{Improved AGB temperatures remove apparent overionisation of Fe I}

\author{
S. W. Campbel1 ${ }^{1,2,3}$, B. T. MacLean ${ }^{2,3}$, V. D’Orazi ${ }^{4}$, L. Casagrande ${ }^{5}$, G. M. de Silva ${ }^{6,7}$, D. Yong ${ }^{5}$, \\ P. L. Cottrell ${ }^{8}$, and J. C. Lattanzio ${ }^{2,3}$
}

\author{
1 Max-Planck-Institut für Astrophysik (MPA), Karl-Schwarzschild-Strasse 1, 85748 Garching, Germany \\ e-mail: scampbell@mpa-garching.mpg.de \\ 2 School of Physics and Astronomy, Monash University, 3800 Clayton, Victoria, Australia \\ 3 Monash Centre for Astrophysics (MoCA), Monash University, 3800 Clayton, Victoria, Australia \\ ${ }^{4}$ INAF-Osservatorio Astronomico di Padova, vicolo dell'Osservatorio 5, 35122 Padova, Italy \\ 5 Research School of Astronomy and Astrophysics, Australian National University, Canberra, ACT 2611, Australia \\ 6 Australian Astronomical Observatory, 105 Delhi Rd, North Ryde, NSW 2113, Australia \\ 7 Sydney Institute for Astronomy, School of Physics, The University of Sydney, NSW 2006, Australia \\ 8 Department of Physics and Astronomy, University of Canterbury, Private Bag 4800, 8140 Christchurch, New Zealand
}

Received 4 May 2017 / Accepted 4 July 2017

\begin{abstract}
Context. A recent study reported a strong apparent depression of Fe I, relative to Fe II, in the AGB stars of NGC 6752. This depression is much greater than that expected from the neglect of non-local thermodynamic equilibrium effects, in particular the dominant effect of overionisation. The iron abundances derived from Fe I were then used to scale all other neutral species in the study.

Aims. Here we attempt to reproduce the apparent Fe discrepancy, and investigate differences in reported sodium abundances.

Methods. We compare in detail the methods and results of the recent study with those of an earlier study of NGC 6752 AGB stars. Iron and sodium abundances are derived using Fe I, Fe II, and $\mathrm{Na}$ I lines. We explore various uncertainties to test the robustness of our abundance determinations.

Results. We reproduce the large Fe I depression found by the recent study, using different observational data and computational tools. Further investigation shows that the degree of the apparent Fe I depression is strongly dependent on the adopted stellar effective temperature. To minimise uncertainties in Fe I we derive temperatures for each star individually using the infrared flux method (IRFM). We find that the $T_{\text {eff }}$ scales used by both the previous studies are cooler, by up to $100 \mathrm{~K}$; such underestimated temperatures amplify the apparent Fe I depression. Our IRFM temperatures result in negligible apparent depression, consistent with theory. We also re-derived sodium abundances and, remarkably, found them to be unaffected by the new temperature scale. $[\mathrm{Na} / \mathrm{H}]$ in the AGB stars is consistent between all studies. Since $\mathrm{Fe}$ is constant, it follows that $[\mathrm{Na} / \mathrm{Fe}]$ is also consistent between studies, apart from any systematic offsets in Fe.

Conclusions. We recommend the use of $(V-K)$ relations for AGB stars, based on comparisons with our individually-derived IRFM temperatures, and their inherently low uncertainties. We plan to investigate the effect of the improved temperature scale on other elements, and re-evaluate the subpopulation distributions on the AGB, in the next paper of this series.
\end{abstract}

Key words. stars: AGB and post-AGB - stars: abundances - stars: evolution - techniques: spectroscopic - globular clusters: general

\section{Introduction}

Due to their relatively homogeneous stellar populations, Galactic globular clusters (GCs) have long been used to constrain stellar evolution models of low-mass stars (e.g. Castellani \& Renzini 1968; Schwarzschild 1970; Iben 1971; Zinn 1974; Norris 1974; Sweigart 1997; Baraffe et al. 1997; Salaris et al. 2016). The colour-magnitude diagrams of GCs generally exhibit wellpopulated sequences corresponding to most phases of stellar evolution. Additionally, most GCs are chemically homogeneous in heavy elements, for example the star-to-star variation of iron is usually smaller than the observational uncertainties. Main sequence observations indicate that the age differences between the subpopulations are undetectable, or small ( $\left.\lesssim 10^{8} \mathrm{yr}\right)$, as compared to total ages of up to 13 Gyr (e.g. Piotto et al. 2007). For most purposes, the stars in each GC can be considered coeval.
In contrast to this homogeneity, observations of the light elements have revealed a consistent picture of subpopulations within each GC. Supported also by photometry, these subpopulations are most clearly seen in multi-dimensional chemical space, for example in the Na-O plane. Indeed Carretta et al. (2010) suggest that a negative/anti-correlation in $\mathrm{Na}-\mathrm{O}$ is the defining feature of a GC, clearly separating them from open clusters which show light element homogeneity (de Silva et al. 2009; MacLean et al. 2015). In addition to the well studied variation in the elements such as $\mathrm{C}, \mathrm{N}, \mathrm{O}, \mathrm{Na}, \mathrm{Mg}, \mathrm{Al}$, there is a growing body of evidence that helium also varies (e.g. D'Antona et al. 2005; Milone 2015; Valcarce et al. 2016). This is qualitatively consistent with proton-capture nucleosynthesis, whereby $\mathrm{H}$ is burned to $\mathrm{He}$ through the $\mathrm{CNO}$ cycle (converting $\mathrm{C}$ and $\mathrm{O}$ to $\mathrm{N}$ ), and $\mathrm{Al}$ and $\mathrm{Na}$ are produced through the $\mathrm{Mg}-\mathrm{Al}$ and $\mathrm{Ne}-\mathrm{Na}$ cycles. We refer the reader to Gratton et al. (2012) for a complete review of multiple populations in GCs. 
Whilst the now well-established existence of multiple subpopulations in GCs adds significant complexity to understanding GCs and their formation, it opens up new opportunities in constraining stellar evolution models since each GC has (at least) two populations practically identical in age and heavy element composition, but different in light element composition. Thus GCs can provide differential comparisons between models of different initial light element constitutions, and in particular, helium content, which is a dominant factor in a star's evolution (e.g. D' Antona et al. 2002; Karakas et al. 2014; Chantereau et al. 2016).

Until recently chemical abundance studies of the GC multiple populations have mainly focused on red giant branch (RGB) stars. Studies of earlier phases of evolution such as the main sequence and sub giant branch have shown that the proportions of stars making up each subpopulation within a GC are generally constant through the colour-magnitude diagram. It has also been shown that the subpopulations occupy different locations on the horizontal branch (HB; see e.g. Marino et al. 2011; Gratton et al. 2015).

The phase of evolution directly after the HB, the asymptotic giant branch (AGB), has only recently started to be investigated systematically. The AGB is particularly interesting because it should contain information about the previous phase, the $\mathrm{HB}$, which is one stage of evolution that is predicted to diverge significantly between He-rich and He-normal stars ${ }^{1}$. Evolutionary models of HB stars are also known to have very substantial uncertainties (e.g. Constantino et al. 2015; Campbell et al. 2016; Constantino et al. 2016). Early low-resolution spectroscopic work on GC giant stars sometimes contained a few AGB stars (usually tentatively identified, see Campbell et al. 2006 for a summary). In some cases these early studies showed possible differences in subpopulation ratios between the AGB and RGB. For example Norris et al. (1981) found a lack of AGB stars with strong cyanogen $(\mathrm{CN})$ band strengths in NGC 6752, as compared to their RGB sample, and Mallia (1978) found a dominance of $\mathrm{CN}$-strong stars on the AGB of 47 Tuc. Cyanogen (roughly) tracks $\mathrm{N}$ content, such that $\mathrm{CN}$-weak stars are first generation/subpopulation (hereafter SP1) and CNstrong stars are second subpopulation (hereafter SP2). These studies were however hampered by low resolution, imprecise photometry (required for separating the RGB and AGB stars), and small samples of AGB stars. Decades later the quality of photometry had improved such that Sneden et al. (2000) and Campbell et al. (2006) argued that it should now be possible to study the AGB stars of GCs in a systematic way. Campbell et al. (2010) presented some early $\mathrm{CN}$ results for a systematic study of AGB stars in $9 \mathrm{GCs}$, based on medium resolution spectra $(R \sim 3000)$. The findings were mixed, with a range of interpretations being possible. This was due to the uncertainties in molecular band formation, which is dependent on temperature, as well as the interrelated abundances of $\mathrm{C}, \mathrm{N}, \mathrm{O}$. One GC did appear to be a clear case though - NGC 6752. Its AGB was dominated by $\mathrm{CN}$-poor giants, in agreement with Norris et al. (1981). Norris et al. (1981) had speculated that this may imply that all of the SP2 stars avoid the AGB phase. This is however not expected from stellar theory - about $50 \%$ of the AGB stars are predicted to be SP2 (CN-strong, Na-rich;

\footnotetext{
1 Due to the more rapid evolution of He-rich stars they have lower stellar masses at a given age. Since the HB core masses do not change significantly between $\mathrm{He}$ populations, the envelope masses on the $\mathrm{HB}$ are reduced, and the $T_{\text {eff }}$ increased, giving rise to blue extensions in the observed HBs.
}

Cassisi et al. 2014). Such a claim of strong discordance between observation and theory required stronger evidence. This was provided by Campbell et al. (2013) with sodium abundance measurements from high-resolution spectroscopy of 24 RGB and 20 AGB stars in NGC 6752. The Na results confirmed the CN results, and Campbell et al. (2013) inferred that all of the Na-rich (SP2) stars were avoiding the AGB phase in NGC 6752.

Since the NGC 6752 study a number of research groups have investigated the AGB stars of many other GCs, with high-resolution spectroscopy - 47 Tuc: Johnson et al. (2015); M2, M3, M5, M13: García-Hernández et al. (2015); M62: Lapenna et al. (2015); M4: MacLean et al. (2016); NGC 2808: Wang et al. (2016); NGC 6752: Lapenna et al. (2016). So far no consistent picture of subpopulation ratios on the AGB has emerged. Interestingly, for the two GCs that have been studied more than once so far, conflicting evidence has been reported. In the case of M4 photometric inferences of population proportions (Lardo et al. 2017) disagree with the spectroscopic results (MacLean et al. 2016). The conflicting spectroscopic evidence for the other case, NGC 6752 (Campbell et al. 2013; Lapenna et al. 2016) is the topic of the current study. Adding to the debate, a photometric study on NGC 6752 AGB stars has very recently been accepted for publication (Gruyters et al. 2017). We refer the reader to MacLean et al. (2016) for a more detailed summary of the literature thus far.

\section{Conflicting results for NGC 6752}

The Campbell et al. (2013) study (hereafter C13) found that the sodium abundances in their sample of NGC 6752 AGB stars were consistent with a single value - the standard deviation of $[\mathrm{Na} / \mathrm{Fe}]$ was $\sigma=0.10$, comparable to the internal errors of $\sim \pm 0.1$ dex. They reported that the single value corresponds to that of the O-rich/Na-poor subpopulation of NGC 6752 (SP1, often referred to as "first generation"). We note that there have been at least three subpopulations identified in NGC 6752, one with field-star-like composition, and the other two with enhanced $\mathrm{Na}$ and reduced $\mathrm{O}$ (amongst other light element variations, see Carretta et al. 2012). For simplicity we divide them here into just two groups: SP1 and SP2.

In contrast to the $\mathrm{C} 13$ result, Lapenna et al. (2016, hereafter L16) report a distinctly different distribution in $[\mathrm{Na} / \mathrm{Fe}]$. In particular they find that about $50 \%$ of their sample have enhanced $[\mathrm{Na} / \mathrm{Fe}]$ - and corresponding (anti-) correlations with $[\mathrm{C}, \mathrm{N}$, $\mathrm{O}, \mathrm{Al} / \mathrm{Fe}$ ] (see their Fig. 2). As they state, this result is much more consistent with current stellar evolution predictions (C13; Cassisi et al. 2014). L16 re-observed the AGB stellar sample of C13 (20 stars) with a different instrument, the UVES spectrograph on the VLT. C13 used data collected using FLAMES (Pasquini et al. 2003), also on the VLT. Thus the spectra and analysis methods are independent, but the AGB stellar samples are identical.

The L16 study did not investigate why the results of the two studies differ so much. The study also did not observe or homogeneously re-analyse RGB stars, which are very useful as a control sample, since they have been well studied in NGC 6752, and they show the full range of $\mathrm{Na}-\mathrm{O}$ dispersion for the particular analysis methodology that one adopts (C13 included 24 RGB stars). Here we explore the methods, uncertainties, and assumptions of both studies with an aim to finding a robust result for $[\mathrm{Na} / \mathrm{Fe}]$. We will investigate other elements in the next paper of the series. We begin by directly comparing the key parameters and results of the two studies. 

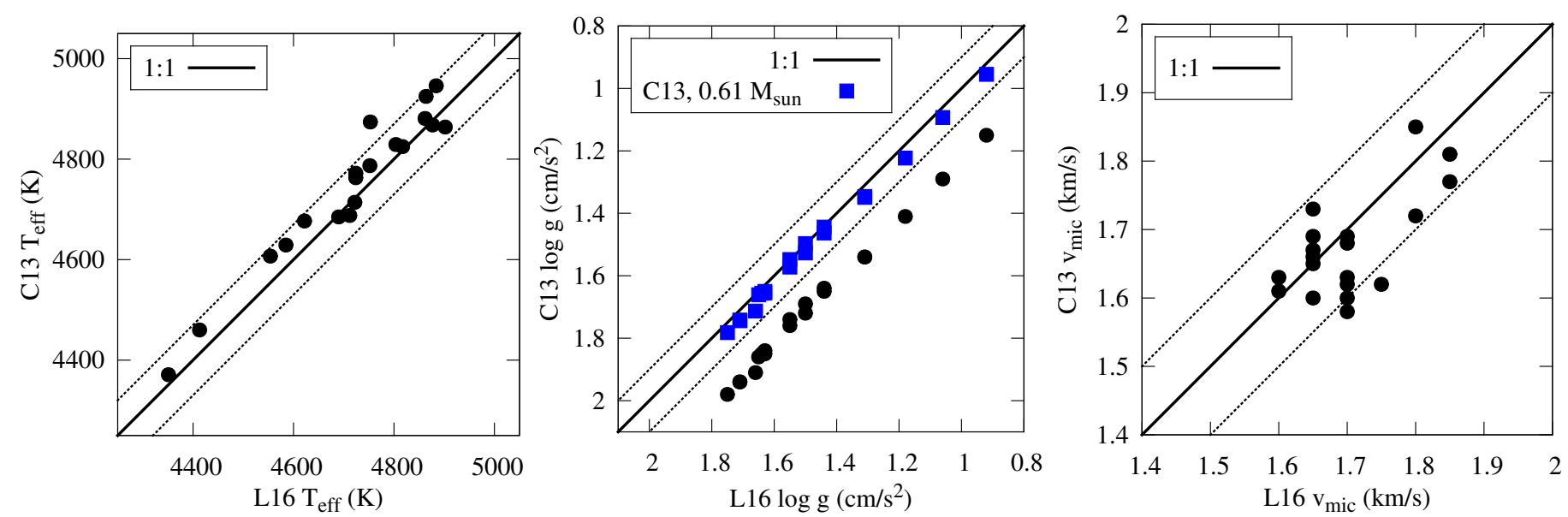

Fig. 1. Comparison of $T_{\text {eff }}($ left $), \log g$ (centre), and $v_{\text {mic }}$ (right) values adopted for the AGB sample by C13 and L16. Dotted lines show typical uncertainties in each of the parameters ( $T_{\text {eff }}$, from colour- $T_{\text {eff }}$ relation: $\pm 70 \mathrm{~K} ; \log g: \pm 0.1 \mathrm{~km} \mathrm{~s}^{-1} ; v_{\text {mic }}: \pm 0.1$ dex). The centre panel also shows our new $\log g$ values calculated using a more appropriate mass estimate for the AGB stars $\left(0.61 M_{\odot}\right.$, blue squares; see text for details).

\section{Comparisons between $\mathrm{C} 13$ and $\mathrm{L} 16$}

\subsection{Stellar parameter comparison}

The stellar parameters - effective temperature $T_{\text {eff }}$, surface gravity $\log g$, microturbulent velocity $v_{\text {mic }}$, and global metallicity $[\mathrm{M} / \mathrm{H}]$ - are central to the spectroscopic determination of abundances. They are the parameters that define the stellar atmosphere model one uses to infer the strength of each line. The parameters are well known to have degeneracies, for example a change in $\log g$ can mimic a change in $[\mathrm{Fe} / \mathrm{H}]$. It is therefore imperative to compare the stellar parameters of $\mathrm{C} 13$ and $\mathrm{L}_{16}{ }^{2}$.

L16 derived $T_{\text {eff }}$ by requiring no trend between iron abundances and excitation potential, which is usually referred to as "spectroscopic" $T_{\text {eff }}$. On the other hand C13 used "photometric" $T_{\text {eff }}$, which is derived from colour- $T_{\text {eff }}$ relations. The left panel of Fig. 1 shows that the $T_{\text {eff }}$ values compare well, with virtually all temperatures being the same within the uncertainties given by the colour- $T_{\text {eff }}$ relations (C13 used the Alonso et al. 1999 relations). It is interesting that there is agreement despite the different methods used to arrive at the final temperatures (although see Sect. 4.2.2).

The centre panel of Fig. 1 shows that there is a constant offset of about 0.2 dex in surface gravity $(\log g)$ between $\mathrm{C} 13$ and L16, with the L16 gravities being lower. This was noted by L16, who suggested that it could be due to the adopted distance modulus or stellar mass. C13 used the same distance modulus as L16 $\left((m-M)_{V}=13.13\right.$; Harris 1996). However C13 neglected to account for mass loss between the RGB and AGB. They adopted the same mass for AGB stars as used for the RGB stars, $0.84 M_{\odot}$, which is clearly incorrect. Following L16 we adjusted the mass for the AGB stars to $0.61 M_{\odot}$, the median HB mass inferred for NGC 6752 by Gratton et al. (2010), and recalculated $\log g$. It can be seen that this removes the offset between L16 and C13, bringing the gravities in to near perfect agreement (blue squares in Fig. 1).

In $\mathrm{C} 13$ the microturbulent velocity $v_{\text {mic }}$ was determined using the relation of Gratton et al. (1996), whilst in L16 it was obtained spectroscopically, by requiring no trend between the reduced equivalent widths and abundances derived from Fe I lines. The right panel of Fig. 1 shows that the L16 values are quantised, in $0.05 \mathrm{~km} \mathrm{~s}^{-1}$ steps. This is most likely due to $0.05 \mathrm{~km} \mathrm{~s}^{-1}$ steps

\footnotetext{
2 These comparisons are in the context of 1D local thermodynamic equilibrium (LTE) abundance analyses.
}

being taken to arrive at a spectroscopic solution, a reasonable approach given the uncertainty in this parameter. The values cover a small range $\left(1.60\right.$ to $\left.1.85 \mathrm{~km} \mathrm{~s}^{-1}\right)$, and the two studies agree considering the characteristic uncertainty of $\pm 0.1 \mathrm{~km} \mathrm{~s}^{-1}$.

With regards to the global metallicity used for atmospheric modelling, L16 used $[\mathrm{M} / \mathrm{H}]=-1.50$ whilst $\mathrm{C} 13$ used $[\mathrm{M} / \mathrm{H}]=$ -1.54 . This is a small difference and is not expected to affect the results significantly.

In summary, apart from the gravity offset, all other stellar parameters show no significant difference between the two studies. Amongst the species under investigation here (Fe I, Fe II, Na I), gravity should mainly affect Fe II. Na I is expected to be largely unaffected ${ }^{3}$ and for this reason we continue with the comparison using the published $\mathrm{C} 13 \mathrm{Na}$ abundances.

\section{2. $[\mathrm{Na} / \mathrm{H}]$ comparison}

To derive abundances of sodium both studies used the equivalent width (EW) method. C13 utilised the strong Na I doublets at 5682/5688 $\AA$ and $6154 / 6160 \AA$, although only the first doublet was usually measurable in the AGB stars. As far as we are aware L16 used the same doublet for their AGB sample (see their Fig. 1). The L16 data has a moderately higher resolution (UVES, $R=40000$ ) than that obtained by C13 (FLAMES, $R=24000$ ).

In Fig. 2 we show the difference between the C13 and L16 $[\mathrm{Na} / \mathrm{H}]$ results $^{4}$. Apart from the two coolest stars, which have lower $\mathrm{Na}$ in L16 (we discuss these stars further in Sect. 6), there is no significant difference in $[\mathrm{Na} / \mathrm{H}]$. There is a slight systematic offset to lower $[\mathrm{Na} / \mathrm{H}]$ in L16 ( - $0.05 \mathrm{dex})$. Adding in the uncertainties from L16, and considering that the uncertainties quoted are internal only, the agreement is remarkable. This strongly suggests that a range of factors have no significant effect on the $\mathrm{Na}$ I abundance derivation, including the following:

- gravity offset in C13 (as expected, see footnote 3); - increase in resolution in L16 over C13;

3 This is due to Na I being the minority species (in these stars sodium is predominantly in the form of $\mathrm{Na} \mathrm{II}$ ) and thus its line formation is insensitive to pressure. Since the atmospheric pressure is primarily determined by gravity, it follows that the formation of $\mathrm{Na}$ I lines is not sensitive to changes in gravity (see e.g. the discussion in Chap. 13 of Gray 2005).

4 We adopt the Grevesse \& Sauval (1998) solar Na value $\log \epsilon=6.33$ for scaling. 


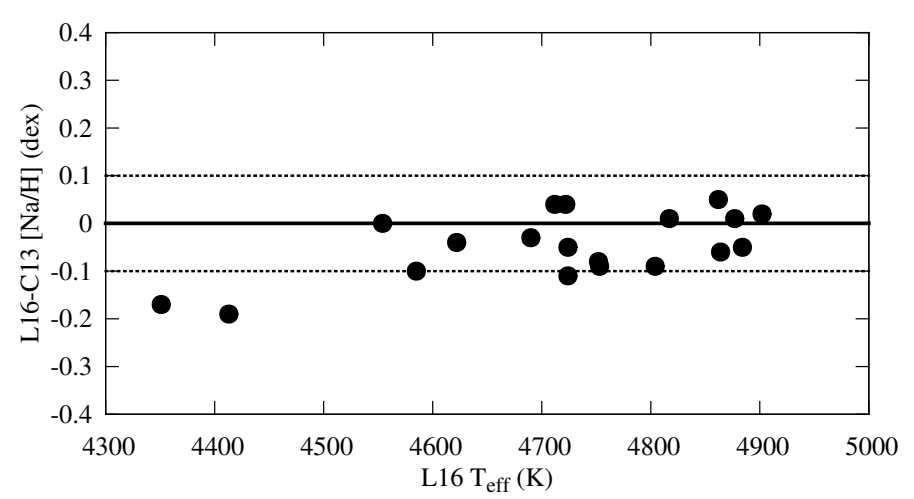

Fig. 2. Difference in $[\mathrm{Na} / \mathrm{H}]$ between the two studies. Dotted lines indicate typical uncertainties.

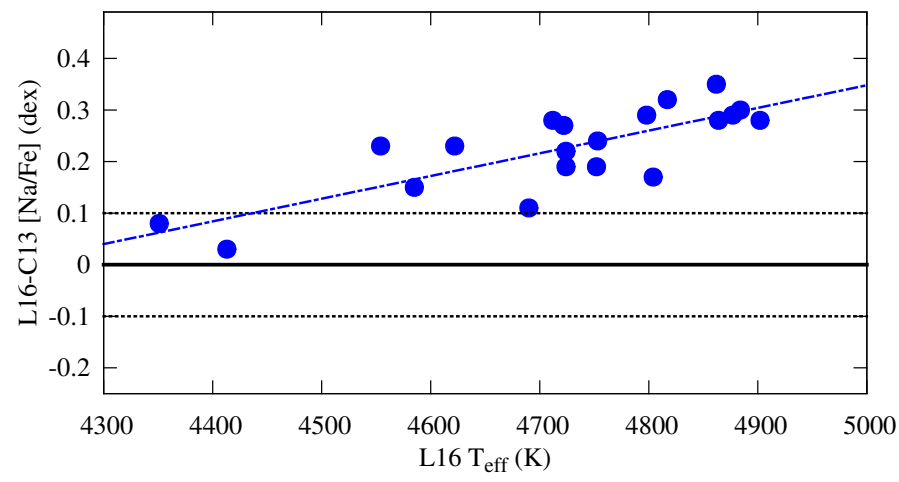

Fig. 3. Difference in $[\mathrm{Na} / \mathrm{Fe}]$ results between $\mathrm{L} 16$ and $\mathrm{C} 13$. The dashdotted line is a linear fit. Differences much larger than the typical uncertainties (dotted lines) are present.

- small differences in model atmospheres and their inputs (e.g. $[\mathrm{M} / \mathrm{H}])$

- small differences between the spectroscopically derived temperatures (L16) and the photometric temperatures (C13);

- scatter in the microturbulent velocities.

The result of this comparison is reassuring and gives confidence in the methods used to derive $[\mathrm{Na} / \mathrm{H}]$. $\mathrm{C} 13$ argued that their $\mathrm{Na}$ results are consistent with a single value, given the uncertainties, and that the value corresponds to SP1 of NGC 6752. This conclusion is however at odds with the L16 study, which concluded that the slightly larger spread found for $[\mathrm{Na} / \mathrm{H}](\sigma=0.13$ dex versus 0.10 dex in $\mathrm{C} 13)$ is significant. Based on the uncertainty estimates of L16 ( $\sim 0.06$ dex, judging from $[\mathrm{Na} / \mathrm{H}]$ in their Fig. 2$)$, which are somewhat smaller that those of C13 ( 0.10 dex), this conclusion may be correct - assuming the L16 error estimates are realistic. We explore various sources of uncertainty in Sects. 4 and 5.2. We now investigate the considerable differences in $[\mathrm{Na} / \mathrm{Fe}]$ between the two studies.

\section{3. $[\mathrm{Na} / \mathrm{Fe}]$ comparison}

In Fig. 3 we show the difference in $[\mathrm{Na} / \mathrm{Fe}]$ between the two studies. Differences of up to +0.35 dex can be seen, although they range from zero to this very high value. Interestingly there is a temperature trend, with stars with the highest $T_{\text {eff }}$ having the largest differences in $[\mathrm{Na} / \mathrm{Fe}]$. A linear regression analysis shows that the Pearson correlation coefficient $r^{2}=0.63$ and that the slope is highly significant $(t$-statistic $=5.6 \sigma)$. This was described in L16 as a systematic offset of 0.25 dex.

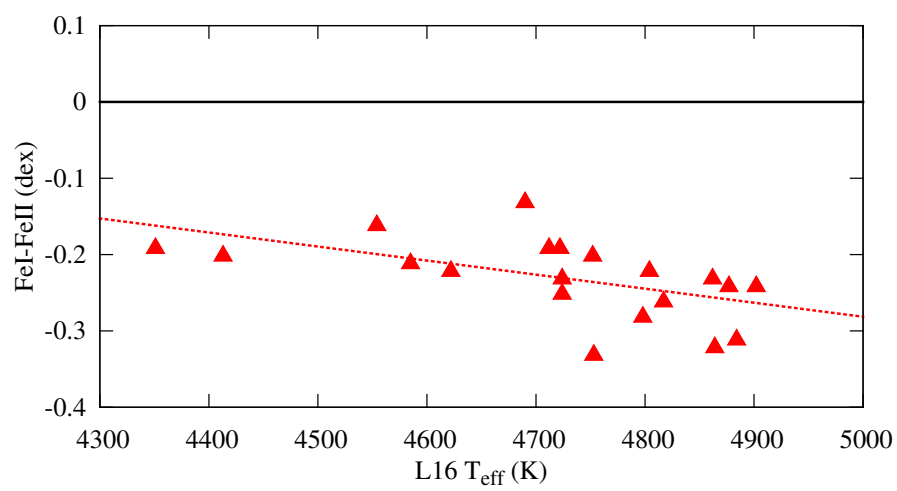

Fig. 4. Depression of Fe I relative to Fe II in the L16 study. The dotted line is a linear fit.

Given our conclusion about $[\mathrm{Na} / \mathrm{H}]$, that the results are practically identical between studies, the $[\mathrm{Na} / \mathrm{Fe}]$ differences must be wholly driven by the denominator, i.e. the Fe distribution must give rise to the difference in $[\mathrm{Na} / \mathrm{Fe}]$ distribution.

With respect to the methodology used to derive $[\mathrm{Na} / \mathrm{Fe}]$ for the NGC 6752 AGB stars the two studies diverge considerably. C13 did not derive Fe abundances. They instead assumed a single $\mathrm{Fe}$ abundance for all stars in their sample $([\mathrm{Fe} / \mathrm{H}]=-1.54$, Carretta et al. 2007). This assumption is discussed further at the beginning of Sect. 3. In contrast, L16 did derive Fe abundances, based on both Fe I and Fe II. An important part of their methodology was that they did not derive $\log g$ spectroscopically, at least not in the common meaning of spectroscopically (we set out the steps in their method in Sect. 3). This was done specifically to avoid "forcing" the abundances of Fe I and Fe II to be equal. To motivate this choice L16 cite some studies that have reported $\mathrm{Fe}$ differences, $\delta \mathrm{Fe}=\mathrm{Fe} \mathrm{I}-\mathrm{Fe}$ II, in globular cluster RGB and AGB stars (Ivans et al. 2001; Lapenna et al. 2014, 2015; Mucciarelli et al. 2015). Certainly not requiring that $\mathrm{Fe} \mathrm{I}=$ $\mathrm{Fe}$ II is necessary for detecting any possible $\delta \mathrm{Fe}$, which would most likely be due to overionisation of Fe I (Lind et al. 2012), but, as we show later (Sect. 4), care is required in order to be confident of the quantitative results. In particular there needs to be a high level of confidence in the stellar parameters used, otherwise an apparent overionisation can be misinterpreted as a real physical phenomenon ${ }^{5}$.

Crucially, to obtain $[\mathrm{Na} / \mathrm{Fe}] \mathrm{L} 16$ decided to use only Fe abundances derived from $\mathrm{Fe}$ I lines in the denominator, following the original suggestion of Ivans et al. (2001; see also Lapenna et al. 2014, 2015; Mucciarelli et al. 2015). Moreover, abundances for all other elements that were determined from neutral species were also scaled by Fe I. We discuss the basis and validity of this choice in Sect. 7.

In Fig. 4 we show the run of $\delta \mathrm{Fe}$ in the L16 data. Apart from the extra scatter added because of the (small, up to $0.03 \mathrm{dex}$ ) differences in Fe II, this shows the same trend as the $[\mathrm{Na} / \mathrm{Fe}]$ differences in Fig. 3. A linear regression analysis shows the slope is significant $(t=2.7 \sigma)$. The $\delta \mathrm{Fe}$ values range up to $\sim-0.35 \mathrm{dex}$. Also of note is that there are no stars with an absolute value of $\delta$ Fe less than 0.1 dex. The L16 Fe abundances are based on many lines and have very small reported uncertainties $( \pm 0.01 \mathrm{dex}$, Table 1 of L16). Thus the entire sample appears to have highly significant $\delta \mathrm{Fe}$. L16 conclude that there is currently no complete explanation of this $\delta \mathrm{Fe}$ effect but it "seems to be a general feature of AGB stars in GCs". This conclusion does however rely

\footnotetext{
5 That is, a physical phenomenon that is not captured by the LTE treat-
} ment. 


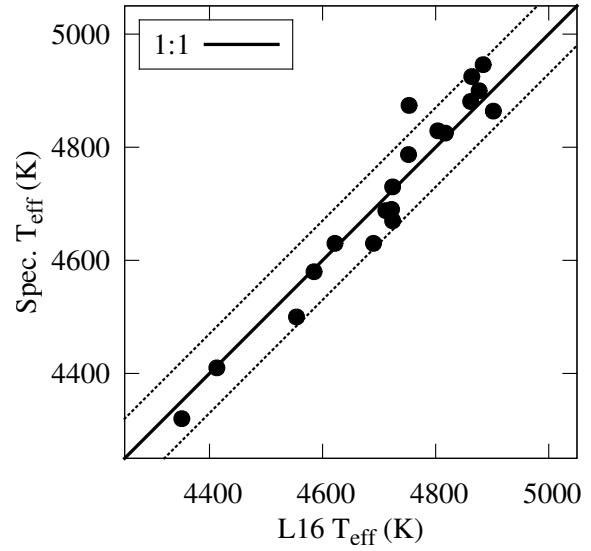

Fig. 5. Spectroscopically determined $T_{\text {eff }}$ for the $\mathrm{C} 13$ data using the L16 method (Sect. 3), compared to the L16 temperatures. The two sets of temperatures were derived using different photometry for initial $T_{\text {eff }}$ estimates. Dashed lines indicate typical uncertainties $( \pm 70 \mathrm{~K})$.

on the reported uncertainties being realistic. We address this fundamental condition in Sects. 4 and 5.2.

In summary, we conclude that the differences in $[\mathrm{Na} / \mathrm{Fe}]$ between L16 and C13 are driven wholly by the Fe I depression relative to Fe II reported by L16.

Our next step in the comparison is to see if we can reproduce the $\mathrm{L} 16 \delta \mathrm{Fe}$ from the C13 FLAMES data.

\section{Fel and Fe II from $\mathrm{C} 13$ data using $\mathrm{C} 13$ parameters}

As noted earlier, C13 did not derive Fe abundances. A single $\mathrm{Fe}$ abundance was assumed for all stars in their sample, based on a detailed RGB study $([\mathrm{Fe} / \mathrm{H}]=-1.54$, Carretta et al. 2007). This was considered a reasonable assumption since it is well established that NGC 6752 is mono-metallic in Fe (Gratton et al. 2005; Carretta et al. 2009; Yong et al. 2015). However it meant that any unexpected deviation in Fe I or Fe II in the AGB stars would have been missed.

Here we present newly calculated Fe I and Fe II abundances using the C13 FLAMES data, in order to compare directly with the $\mathrm{L} 16 \mathrm{Fe}$ results.

We derive LTE Fe abundances using the EW method, as in the L16 study. While C13 used the MOOG stellar line analysis program (Sneden 1973), here we use the WIDTH3 program (Gratton 1988; Gratton \& Sneden 1988). We aim to reproduce the L16 results, so we follow the specific methodology of that study, which comprises the following steps:

1. $T_{\text {eff }}$ is calculated "spectroscopically", i.e. by requiring no trend between Fe I abundances and excitation potential.

2. Gravity is adjusted from the initial photometric values by recalculating it using the $T_{\text {eff }}$ from Step 1. Iteration back to Step 1 may be required if the changes in $\log g$ are significant. Ionisation balance is ignored.

3. Microturbulent velocity is then adjusted by requiring no trend between Fe I abundances and line strengths.

We used Kurucz (1993) model atmospheres, adopting the same $[\mathrm{M} / \mathrm{H}]=-1.50$ value as L16. Photometrically-derived values of $T_{\text {eff }}$ and $\log g$ were adopted as initial estimates (those in Fig. 1). The initial temperatures are identical to those used in C13, based on the Strömgren photometry from Grundahl et al. (1999) and

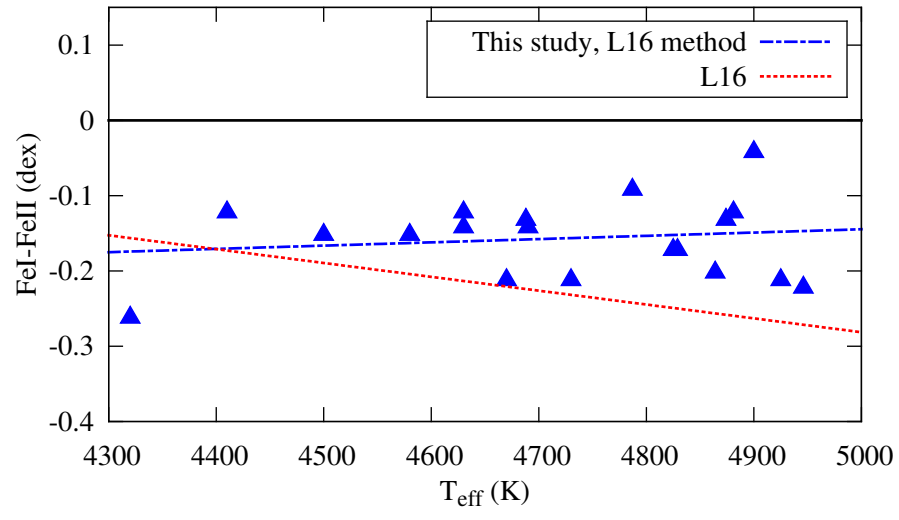

Fig. 6. Depression of Fe I relative to Fe II we find when using the method of L16 and the C13 data (blue triangles). The blue dot-dashed line is a linear fit to our results, and the dashed red line is the fit to the L16 results (from Fig. 4). The temperatures used for this analysis are displayed in Fig. 5.

using the $(b-y)$ relation of Alonso et al. (1999, their Eq. (15)). NGC 6752 suffers from minor reddening; we corrected the $b$ and $y$ magnitudes for reddening using the relations of Schlegel et al. (1998), adopting $E(B-V)=0.04$ mag (Harris 1996). The initial $\log g$ values (blue squares in Fig. 1) were calculated using a stellar mass of $0.61 M_{\odot}$, and a distance modulus of $(M-m)_{V}=13.13$ (Harris 1996), consistent with L16. We used the bolometric correction relation of Alonso et al. (1999, their Eqs. (17) and (18)).

Using Step 2 above for gravity estimation one avoids Fe I being forced to be equal to Fe II (i.e. ionisation balance is not enforced). In iterating back to Step 1 we found that the $\log g$ values are insensitive to the $\sim 0 \rightarrow 100 \mathrm{~K}$ modifications in $T_{\text {eff }}$, with the average change in $\log g$ being $\sim+0.03$ dex. Our initial microturbulence values were estimated using the relation from Gratton et al. (1996). Most of these values were unchanged in Step 3, with four AGB stars changing by $\sim \pm 0.1 \mathrm{~km} \mathrm{~s}^{-1}$, so they are still consistent with those in the right panel of Fig. 1. Our final spectroscopic $T_{\text {eff }}$ values are consistent with the L16 spectroscopic temperatures (Fig. 5). Finally, our linelist is based on that of Gratton et al. (2003). We explore line list differences in Sect. 5.2.1.

With these parameters, and assuming a solar abundance for Fe of $\log \epsilon=7.50$ (Grevesse \& Sauval 1998), we find for the AGB stars:

$[\mathrm{Fe} \text { II } / \mathrm{H}]_{\mathrm{AGB}}=-1.48 \pm 0.01 \mathrm{dex}(\sigma=0.04 ; \mathrm{L} 16:-1.58 \mathrm{dex})$

$[\mathrm{Fe} \mathrm{I} / \mathrm{H}]_{\mathrm{AGB}}=-1.63 \pm 0.01 \operatorname{dex}(\sigma=0.04 ; \mathrm{L} 16:-1.80 \mathrm{dex})$.

Thus we confirm a significant Fe I-Fe II difference, at least qualitatively. Unlike the L16 $\delta \mathrm{Fe}$ results our results show no substantial trend with $T_{\text {eff }}$ (Fig. 6), with the significance of the slope being $<1 \sigma$, and $r^{2}=0.03$. The average value of the offset in our results is $\delta \mathrm{Fe}=-0.15 \pm 0.01 \mathrm{dex}(\sigma=0.05)$, as compared to -0.22 dex in L16. Using the C13 parameters and the L16 spectra, L16 found an average offset of -0.27 dex. Thus there is a systematic difference of order 0.1 dex between the studies even if using the same stellar parameters. This may be related to the 0.1 dex lower $[\mathrm{Fe} \mathrm{II} / \mathrm{H}]$ found by $\mathrm{L} 16$, which could be due to the adoption of different oscillator strengths between the studies (we explore this as an uncertainty in Sect. 5.2.1). 


\section{The origin of $\delta \mathrm{Fe}$}

The qualitatively similar finding of a definite $\delta \mathrm{Fe}$ in both sets of data is in one way reassuring - it shows that, given a particular methodology, the results of L16 are reproducible with independent data and tools. However, the L16 study did not investigate the robustness of this result. An obvious question arises - is there some systematic problem(s) in the method mimicking this phenomenon?

To investigate this possibility we explore the uncertainties in the abundance analysis process. We begin by noting that it is well known that (i) offsets in Fe II can be caused by offsets in gravity; (ii) offsets in Fe I can be caused by offsets in $T_{\text {eff }}$; and (iii) the magnitude of non-LTE (NLTE) effects is predicted to be small in these stars. We explore the first two sources of uncertainty in the next two subsections and the third in Sect. 5.2.2.

\subsection{Gravity check: Fe II abundance comparison with RGB stars}

One way to check if there is a gravity offset problem is to compare the Fe II abundance of the AGB stars to that of the RGB stars - they should be identical for Fe II since NLTE effects are predicted to be negligible for Fe II in late-type stars (e.g. Lind et al. 2012). Due to its dependence on gravity, a difference in Fe II may indicate systematic problems with $\log g$ that would require investigation.

C13 included RGB stars in their study, as a control sample. For the Fe determination in the RGB stars we again used the same methodology of L16, as described for the AGB sample above. In this case our results show no evidence of an Fe I-Fe II discrepancy:

$[\mathrm{Fe} \text { II } / \mathrm{H}]_{\mathrm{RGB}}=-1.47 \pm 0.01 \mathrm{dex}(\sigma=0.06)$

$[\mathrm{Fe} \mathrm{I} / \mathrm{H}]_{\mathrm{RGB}}=-1.48 \pm 0.04 \operatorname{dex}(\sigma=0.06)$.

Formally we measure $\delta \mathrm{Fe}=-0.01 \pm 0.02(\sigma=0.08)$.

Importantly, the AGB $[\mathrm{Fe} I \mathrm{II} / \mathrm{H}]$ is perfectly in agreement with the RGB measurement. This suggests that Fe II is not the source of the AGB $\delta \mathrm{Fe}$ phenomenon. Although not a definitive proof, it also suggests that the AGB $\log g$ values are reasonable. Assuming this is correct we now investigate the sensitivity of Fe to $T_{\text {eff }}$.

\subsection{Temperature check: Fe I behaviour with varying $T_{\text {eff }}$}

L16 derived surface temperatures spectroscopically, i.e. by requiring no trend between iron abundances and excitation potential. In this procedure it is usual to use photometric $T_{\text {eff }}$ as an initial estimate. L16 did not specify if this was done, but we assume it was. We also assume the same BV photometry (Stetson 2000) that was used for the $\log g$ derivation was also used for $T_{\text {eff }}$. Regardless of the source of photometry, and the method to arrive at the final temperatures, it can be seen in Fig. 1 that the L16 temperatures agree with the C13 temperatures, within the uncertainties. Here we explore the uncertainties, to ascertain whether systematic problems with $T_{\text {eff }}$ could be giving rise to the $\delta \mathrm{Fe}$ phenomenon present in both studies (Figs. 4 and 6).

\subsubsection{AGB star test case}

To investigate the sensitivity of Fe I and Fe II to the adopted $T_{\text {eff }}$ we chose one star as a case study: AGB star 97. This star was

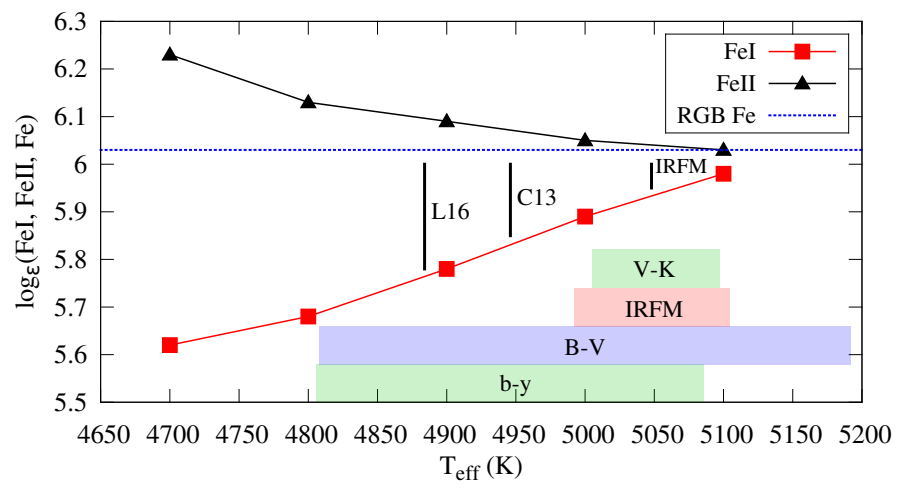

Fig. 7. Testing the effect of adopted $T_{\text {eff }}$ on the derived Fe I and Fe II abundances in the AGB star 97 . Horizontal bars show the $2 \sigma$ uncertainty ranges ${ }^{6}$ for each of the $T_{\text {eff-colour relations, centred on the } T_{\text {eff }} \text { predic- }}$ tion of each relation (see text for details). The temperatures used in the C13 study $(4946 \mathrm{~K})$ and the L16 study $(4884 \mathrm{~K})$ are indicated by vertical lines, highlighting the magnitude of $\delta \mathrm{Fe}$ at each $T_{\text {eff }}$. Also shown is our IRFM $T_{\text {eff }}(5048 \mathrm{~K})$ and associated uncertainty. The $(V-K) T_{\text {eff }}$ is $5051 \mathrm{~K}$. The dashed line shows the average iron abundance of the RGB stellar sample.

chosen because it displays a strong $\delta \mathrm{Fe}$ signal in both L16 and the current study, with $\delta \mathrm{Fe}=-0.31$ and -0.22 dex, respectively. In L16 the adopted surface temperature for this star was $4884 \mathrm{~K}$. In the current study we found the photometric $T_{\text {eff }}$ of $\mathrm{C} 13$ to require no change ( $4946 \mathrm{~K})$. The difference of $62 \mathrm{~K}$ is within the $1 \sigma( \pm 70 \mathrm{~K})$ uncertainties of the Strömgren relation which we used to derive $T_{\text {eff }}$ (Alonso et al. 1999).

For the test we varied $T_{\text {eff }}$ and attempted to find spectroscopic "solutions" (i.e. no trend between iron abundances and excitation potentials) at each $T_{\text {eff. }}$. During this process $\log g$ was kept constant, at the photometric value. In Sect. 3 we showed that the $\log g$ adjustment is negligible within the $T_{\text {eff }}$ uncertainty ranges considered here.

In Fig. 7 we show the results of the test. Interestingly, we were able to find spectroscopic "solutions" for a wide range of temperatures, even outside the uncertainties of the colour- $T_{\text {eff }}$ relations, although no solution was found above $5100 \mathrm{~K}^{7}$. Multiple solutions were possible because of the uncertainty in the abundance-excitation potential slope, combined with the poorly constrained microturbulence parameter, which was adjusted to reduce the slope in the usual procedure (Sect. 3). The slope uncertainty in this case was $\pm 0.03 \mathrm{dex} / \mathrm{eV}^{8}$. Over the $T_{\text {eff }}$ range of $4800 \rightarrow 5100 \mathrm{~K}$ the range of microturbulence values we found spanned $1.20 \rightarrow 1.65 \mathrm{~km} \mathrm{~s}^{-1}$, with the microturbulent velocity increasing with temperature. Apart from the very low $T_{\text {eff }}$ end, which is very unlikely to be representative of the true temperature (Sect. 4.2.3), these appear to be reasonable values, as compared to those reported by C13 and L16 (Fig. 1). It is also useful to remember that "microturbulent velocity" is essentially a free parameter, i.e. it has little physical basis (see e.g. the four listed points in Sect. 1 of Mucciarelli 2011, and references therein). For all solutions there was no trend between Fe abundances and line strength, within the uncertainties.

\footnotetext{
6 We use $2 \sigma$ uncertainties due to the fact that AGB stars are not significantly represented in the stellar samples on which the colour- $T_{\text {eff }}$ relations are based.

7 Within our test procedure. Varying $\log g$ may allow solutions at higher $T_{\text {eff }}$.

8 Across the AGB sample the average uncertainty was $\pm 0.02 \mathrm{dex} / \mathrm{eV}$.
} 
The $\delta \mathrm{Fe}$ variation over the $T_{\text {eff }}$ test range shows a consistent trend: $\delta \mathrm{Fe}$ decreases with increasing $T_{\text {eff }}$. Ignoring the $T_{\text {eff }}$ values outside the photometric $T_{\text {eff }}$ uncertainties, $\delta \mathrm{Fe}$ ranges from -0.46 (at $4820 \mathrm{~K}$ ) to -0.05 (at $5100 \mathrm{~K}$ ). This final value is consistent with zero given the $1 \sigma$ scatter in $\delta \mathrm{Fe}$ of 0.08 dex that we found in the RGB sample (Sect. 3).

Also marked in Fig. 7 are the temperatures used by $\mathrm{C} 13$ and L16. Importantly the $\delta \mathrm{Fe}$ value at the L16 $T_{\text {eff }}$ is very similar to that reported by L16 (their -0.31 dex versus -0.34 dex here). Considering that different spectra and tools have been used, and that both the L16 and $\mathrm{C} 13 \delta \mathrm{Fe}$ values fit the $\delta \mathrm{Fe}-T_{\text {eff }}$ trend, this is a strong confirmation of the $\delta \mathrm{Fe}$ phenomenon, and its dependence on $T_{\text {eff }}$, both qualitatively and quantitatively.

The gradient $\delta \mathrm{Fe} / T_{\text {eff }}$ is $\sim 0.002 \mathrm{dex} / \mathrm{K}$. Given a typical $1 \sigma$ $T_{\text {eff }}$ uncertainty of $\pm 100 \mathrm{~K}$ for the $(B-V)$ colour, this translates to a possible $\delta \mathrm{Fe}$ range of $0.40 \mathrm{dex}$. This is a very substantial uncertainty and consistent with the up to 0.35 dex found by L16.

\subsubsection{Ramifications of the $\delta \mathrm{Fe}$ dependence on $\mathrm{T}_{\text {eff }}$}

This result shows clearly that significant $\delta \mathrm{Fe}$ values can arise even within the photometric $T_{\text {eff }}$ uncertainties. Crucially it appears that the initial $T_{\text {eff }}$ estimate (usually photometric) is central in determining the final $\delta \mathrm{Fe}$ value. This is because there is a continuum of spectroscopic "solutions" across the $T_{\text {eff }}$ uncertainty range, so that the spectroscopically determined $T_{\mathrm{eff}}$ will usually be close to the photometric estimate. Figure 7 then implies that, if there is a systematic trend or offset in the inferred photometric temperatures, a similar trend or offset should be present in $\delta \mathrm{Fe}-$ even if the temperatures are determined spectroscopically. Given this, an investigation into the sources of the adopted temperatures is mandatory, and is our next step.

\subsubsection{Temperature scales and the case for $(\mathrm{V}-\mathrm{K})$}

In Fig. 7 we also show the predictions of three colour- $T_{\text {eff }}$ relations for our AGB test star: Strömgren $(b-y)$ (Alonso et al. 1999 Eq. (15), with a quoted uncertainty of $1 \sigma=70 \mathrm{~K})$, Johnson $(B-V)$ (Alonso et al. 1999 Eq. (4), $\sigma=96 \mathrm{~K}$ ), and Johnson2MASS $\left(V-K_{\mathrm{s}}\right.$ ) (Table 5 of González Hernández \& Bonifacio $2009, \sigma=23 \mathrm{~K})$. Reddening was corrected for in $\left(V-K_{\mathrm{s}}\right)$ using the relation of Fitzpatrick \& Massa (2007, their Eq. (8)) assuming $R_{V}=3.1$ and $E(B-V)=0.04$.

As a cross-check we have also calculated our own $T_{\text {eff }}$ for this star using the Casagrande et al. (2010) implementation of the infrared flux method (IRFM). The IRFM estimates $T_{\text {eff }}$ by comparing the ratio of the observed bolometric flux to a monochromatic IR flux with the ratio predicted by theory (synthetic spectra). Since the synthetic spectra have a very mild dependency on stellar parameters in the IR, this method is only weakly dependent on the models. The Casagrande et al. (2010) scale is calibrated absolutely, using a set of solar twins. For further details of our IRFM procedure we refer the reader to Casagrande et al. (2010). We used the 2MASS JHK (Skrutskie et al. 2006) and BV photometry for this $T_{\text {eff }}$ determination (and for all the IRFM temperatures in this study). The temperature we derived has an internal uncertainty of $\pm 30 \mathrm{~K}$, and is also included in Fig. 7. The BV photometry we use in this study is from Momany et al. (2002). These data are of high quality, for example the average error on the $\mathrm{V}$ magnitudes for the AGB sample is $0.008 \mathrm{mag}$.

Immediately obvious from Fig. 7 is that the $(V-K)$ relation gives the most precise $T_{\text {eff }}$ estimate. It is also in perfect agreement with our IRFM-derived $T_{\text {eff }}$, which has a similar degree of

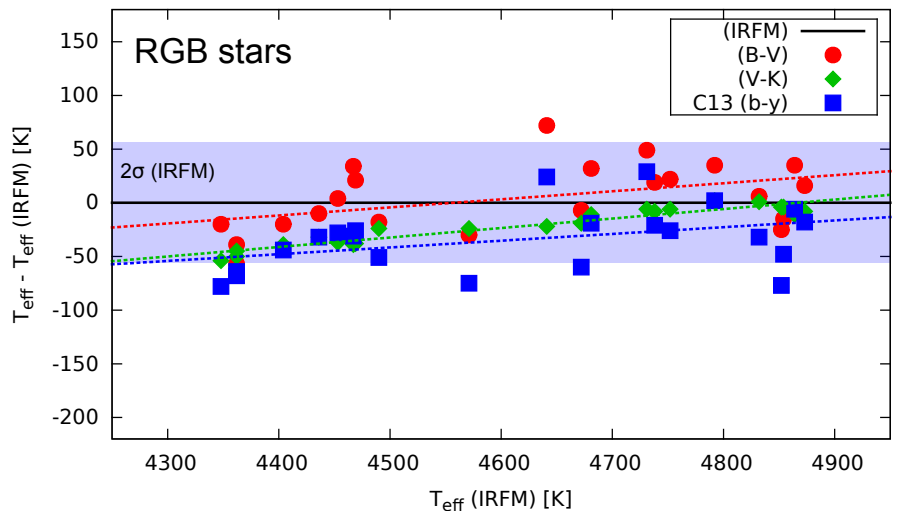

Fig. 8. Comparison of $T_{\text {eff }}$ derived for the RGB stars using four different methods: three different colour- $T_{\text {eff }}$ relations and our IRFM (see text for details). The $T_{\text {eff }}$ from the colour- $T_{\text {eff }}$ relations are shown as differences from the IRFM temperatures. Dashed lines are linear fits to each $T_{\text {eff }}$ set, and the shaded area shows the average $2 \sigma$ internal uncertainty of the IRFM scale $( \pm 56 \mathrm{~K})$. The $2 \sigma$ uncertainties of the $(b-y)$ and $(B-V)$ relations are much larger than for our IRFM, being \pm 140 and $\pm 192 \mathrm{~K}$, respectively (Alonso et al. 1999). The $(V-K)$ uncertainties ( $2 \sigma=46 \mathrm{~K}$; González Hernández \& Bonifacio 2009) are similar to the IRFM uncertainties.

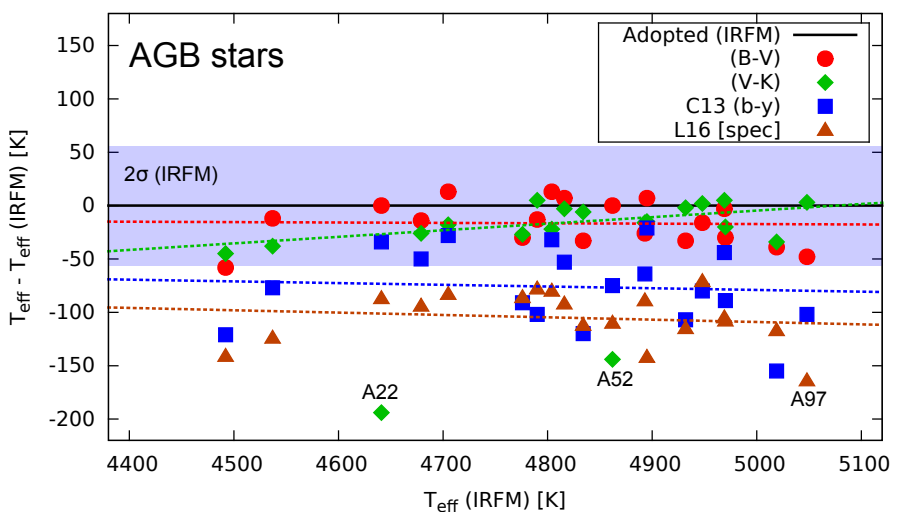

Fig. 9. Comparison of five $T_{\text {eff }}$ determinations for the AGB star sample. Symbols and shading are the same as Fig. 8, except for the addition of the L16 temperatures (L16 did not study RGB stars). Three stars are highlighted with labels: AGB star 97 was the subject of our $\delta$ Fe tests (Fig. 7), whilst A22 and A52 have uncertain IRFM and $(V-K) T_{\text {eff }}$ due to suspect 2 MASS K magnitudes. These latter two stars have not been included in the linear regression lines for $(V-K)$ or IRFM, and we adopt the $(B-V)$ temperatures for them.

precision. Interestingly, both of these $T_{\text {eff }}$ estimates give much lower $\delta \mathrm{Fe}$ values than obtained using either the C13 or L16 temperatures, with $\delta \mathrm{Fe}$ approaching zero at the higher end of the $2 \sigma$ uncertainty bands.

That $(V-K)$ has a small uncertainty for late-type stars is well known and is due to it being (i) only marginally sensitive to metallicity/line blanketing, and (ii) having a negligible dependence on surface gravity (Alonso et al. 1999; Ramírez \& Meléndez 2005). Importantly for our study, the ( $V-$ $K$ ) colour is particularly suited to giants. Indeed Alonso et al. (1999) suggest that it is "probably the best temperature indicator for giant stars". Furthermore, Ramírez \& Meléndez (2005) report that, due to the colour being so insensitive to gravity, particularly in the range $4800 \mathrm{~K}>T_{\text {eff }}>6000 \mathrm{~K}$, it makes $(V-K)$ suitable for stars of unknown luminosity class. This is important for studies of (early) AGB stars because many of them lie in this $T_{\text {eff }}$ range (our sample: $4500 \rightarrow 5050 \mathrm{~K}$ ) and it is a class of 
stars that have only recently started to be investigated in detail, so their surface gravities are less certain than RGB star gravities.

\subsubsection{Temperature scales: ensemble comparisons}

As a further check of the $T_{\text {eff }}$ "scales" we now perform ensemble comparisons between the $T_{\text {eff }}$ predictions from the same three colour- $T_{\text {eff }}$ relations detailed above but across our entire NGC 6752 RGB and AGB samples. We also present our IRFM temperatures for all the stars.

The RGB sample comparison is displayed in Fig. 8. Although small offsets and small temperature trends are present, it is clear that all three relations give temperatures that are consistent with each other, within the quoted $1 \sigma$ uncertainties (Alonso et al. 1999; González Hernández \& Bonifacio 2009). This is true across the whole $T_{\text {eff }}$ range of our RGB sample. This confirms the well-constrained nature of the parameters for GC RGB stars, as expected from much previous work on these types of stars.

The AGB on the other hand has not been well studied. In Fig. 9 we show $T_{\text {eff }}$ for the AGB stars. Here the $(B-V),(V-K)$, and IRFM temperatures are consistent with each other, similar to the RGB case. However the Strömgren $(b-y)$ temperatures (used by $\mathrm{C} 13$ ) are offset by about $-60 \mathrm{~K}$. This is particularly true at higher $T_{\text {eff }}(>4700 \mathrm{~K})$, where the majority of the temperatures are outside the $2 \sigma$ uncertainties of the IRFM $T_{\text {eff }}$. Also displayed are the temperatures from L16. These are offset even more, by about $-100 \mathrm{~K}$ on average.

Given that we have showed in Fig. 7 that $\delta \mathrm{Fe}$ is is strongly correlated with a reduction in $T_{\text {eff }}$, this is very suggestive that the large $\delta \mathrm{Fe}$ reported by L16 (Fig. 4) is driven by the $T_{\text {eff }}$ scale of that study. It also explains our own finding of significant $\delta \mathrm{Fe}$ using the Strömgren $(b-y)$ temperatures adopted by C13. That the temperature scale of C13 is slightly warmer than that of L16 also shows why our $\delta \mathrm{Fe}$ values are generally smaller in magnitude than those of L16 (Sect. 3; Fig. 6).

The next logical step is to use the more appropriate temperatures in deriving Fe abundances. The change in $T_{\text {eff }}$ scale may also affect $\mathrm{Na}$ I, which we also re-derive in Sect. 6.

\section{Iron from $\mathrm{C} 13$ data using new $T_{\text {eff }}$ scale}

\subsection{Reanalysis method and results}

In our final reanalysis of the C13 spectra we chose to use photometric parameters (IRFM) only, because (i) the temperature scale appears quite accurate so we want to avoid additional uncertainties by using the spectroscopic $T_{\text {eff }}$ method; and (ii) following L16, we do not want to force Fe I = Fe II by obtaining $\log g$ spectroscopically. We adopt the same distance modulus as $\mathrm{C} 13$ and L16, a mass of $0.78 M_{\odot}$ for RGB stars and $0.61 M_{\odot}$ for AGB stars. Microturbulent velocities were estimated using the Gratton et al. (1996) relation. Our final stellar parameters are plotted in the $\log g-T_{\text {eff }}$ plane in Fig. 10, and listed in Table A.1. Note that there are 19 AGB stars rather than 20, since for one star we did not have all three sets of photometry (star 89 of C13 and L16).

In Fig. 11 we show the final iron results for our whole sample of RGB and AGB stars. Fe I and Fe II are shown separately for each star. Immediately obvious in this figure is that all abundances fall within the expected uncertainty range characterised by $1 \sigma \sim 0.1$ dex. Final Fe abundances are also listed in Table A.1.

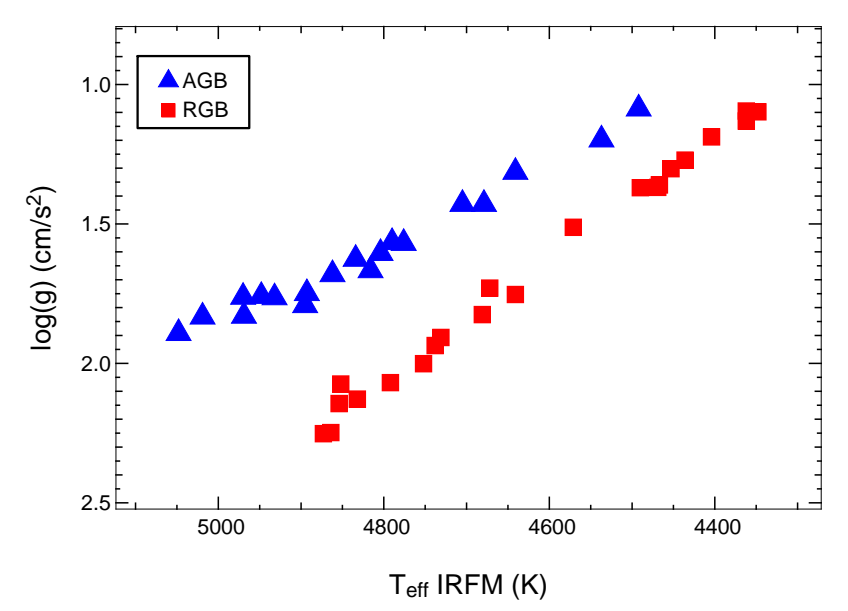

Fig. 10. Our final stellar parameters for the NGC 6752 AGB and RGB stars. All are "photometric" - based on the IRFM temperatures calculated for this study, except for 2 AGB stars (see Fig. 9).

The main effect of the new stellar temperatures is to raise the Fe I values in the AGB sample, as expected from Figs. 7-9. Table 1 shows that the average increase in Fe I is $+0.11 \mathrm{dex}$, as compared to our results using the C13 stellar parameters (Sect. 3). Fe II is unchanged, so this translates directly into a reduction of average $\delta \mathrm{Fe}$, reducing it from -0.15 to -0.04 dex. Figure 12 shows visually that $\delta \mathrm{Fe}$ in the AGB sample is now negligible. A weak trend appears to be visible though, with $\delta \mathrm{Fe}$ increasing in magnitude in the hotter stars $\left(T_{\text {eff }}>4800 \mathrm{~K}\right)$. The average $\delta \mathrm{Fe}$ is however only about -0.1 dex in this subset of AGB stars, and the trend mostly lies within the error band. We speculate that this possible trend may be due to either residual underestimation of $T_{\text {eff }}$, or due to NLTE effects being stronger in the hotter AGB stars (although the latter is not supported by current theory, see Sect. 5.2.2).

The main feature in the average Fe I and Fe II values (Table 1, final set) is that Fe I is lower in the AGB stars than in the RGB stars, by 0.11 dex. This difference is just within the combined $1 \sigma$ dispersions of each sample ( 0.05 and 0.06 , respectively; Table 1$)$, so it is marginally significant. The difference becomes even less significant when considering other uncertainties (Sect. 5.2).

Averaging $\mathrm{Fe}$ abundances from Fe I and Fe II in the AGB and RGB samples to arrive at total $[\mathrm{Fe} / \mathrm{H}]$ values shows that the difference between the two evolutionary phases is $-0.06 \mathrm{dex}$, which is comparable to the star-to-star scatter. Visually the closeness of all the Fe determinations can be seen in Fig. 11.

We also computed abundances for the AGB stars using the $(V-K)$ temperatures. We found this temperature scale to give identical $\delta \mathrm{Fe}$ to the IRFM scale. This was expected since the temperatures are very similar, as seen in Fig. 9. We now explore other uncertainties in the method.

\subsection{Sensitivity of Fe to other uncertainties}

\subsubsection{Weighted oscillator strengths}

The weighted oscillator strength $(\log g f)^{9}$ adopted for each line is a known source of uncertainty in spectroscopic abundance determination (e.g. Gray 2005). Since oscillator strength quantifies the transition probability of a species from one level to the next, a change in $\log g f$ has a systematic effect on derived abundances, 9 Where $f$ is the oscillator strength and $g$ is the statistical weight of the
lower level. 
Table 1. Summary of iron abundances derived from Fe I \& Fe II using different input parameters.

\begin{tabular}{l|cc|cc|cc}
\hline \hline Analysis run & $\begin{array}{c}{[\mathrm{Fe} \mathrm{I} / \mathrm{H}]} \\
(\mathrm{dex})\end{array}$ & $\begin{array}{c}\sigma \\
(\mathrm{dex})\end{array}$ & $\begin{array}{c}{[\mathrm{Fe} \mathrm{II} / \mathrm{H}]} \\
(\mathrm{dex})\end{array}$ & $\begin{array}{c}\sigma \\
(\mathrm{dex})\end{array}$ & $\begin{array}{c}\delta \mathrm{Fe} \\
(\mathrm{dex})\end{array}$ & $\begin{array}{c}\sigma \\
(\mathrm{dex})\end{array}$ \\
\hline AGB L16 & -1.80 & 0.05 & -1.58 & 0.02 & -0.22 & 0.05 \\
\hline RGB (C13 $\left.T_{\text {eff }}\right)$ & -1.48 & 0.06 & -1.47 & 0.06 & -0.01 & 0.08 \\
AGB (C13 $\left.T_{\text {eff }}\right)$ & -1.63 & 0.04 & -1.48 & 0.04 & -0.15 & 0.05 \\
\hline RGB (L16 gfs) & -1.43 & 0.05 & -1.52 & 0.05 & +0.09 & 0.07 \\
AGB (L16 gfs) & -1.52 & 0.06 & -1.53 & 0.04 & +0.01 & 0.08 \\
\hline RGB (Final) & -1.43 & 0.05 & -1.47 & 0.06 & +0.04 & 0.07 \\
AGB (Final) & -1.52 & 0.06 & -1.48 & 0.04 & -0.04 & 0.07 \\
\hline
\end{tabular}

Notes. Abundances are scaled based on a solar Fe abundance of $\log \epsilon=7.50$ dex. Also shown is the difference $\delta \mathrm{Fe}=\mathrm{Fe} \mathrm{I}-\mathrm{Fe}$ II. The first line shows the L16 AGB results. The second set of results were obtained using the C13 temperatures (Sect. 3). The 3rd set of results were obtained using our new IRFM $T_{\text {eff }}$ scale but adopting the L16 $\log g f$ values. Our final results, using the IRFM temperatures and our $\log g f$ values, are in the last two rows. The typical number of Fe I lines analysed was 20-40, and 2-3 for Fe II. The $\sigma$ values are the $1 \sigma$ star-to-star scatter only.

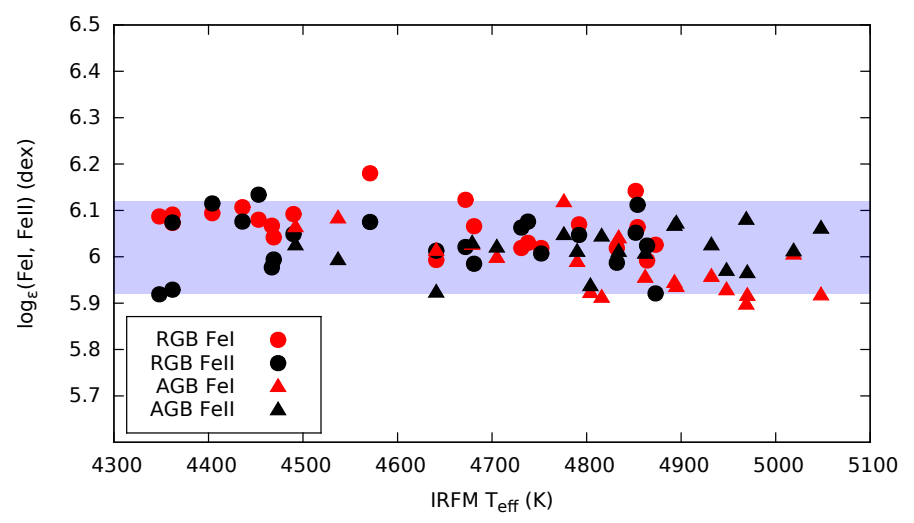

Fig. 11. Iron results using our new IRFM temperatures. All RGB stars (dots) and AGB stars (triangles) are shown for comparison, with iron abundances derived using Fe I and Fe II highlighted by colour (red and black respectively). The shaded region indicates a typical uncertainty of $\sim \pm 0.10$ dex, centered around the average Fe II abundance $(\log \epsilon=6.02$, from RGB and AGB stars).

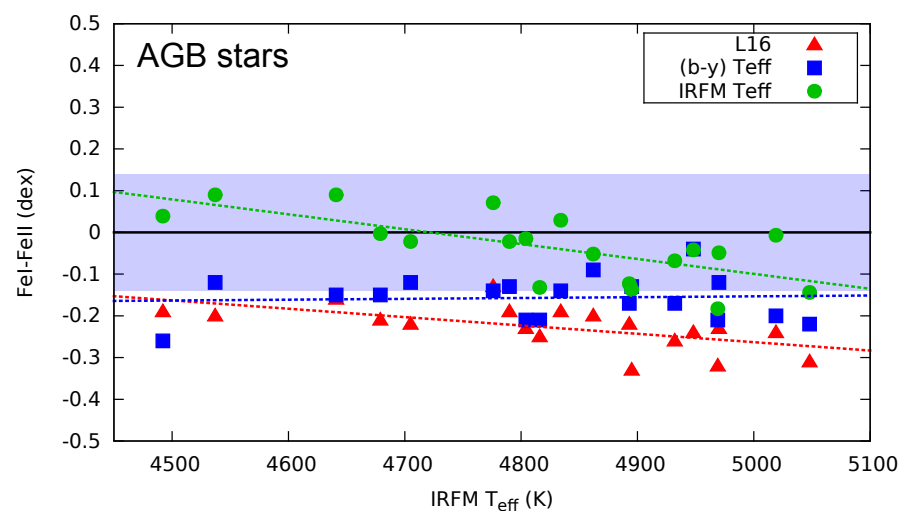

Fig. 12. AGB $\delta \mathrm{Fe}=\mathrm{Fe} \mathrm{I}-\mathrm{Fe}$ II results for the current study obtained using our IRFM temperatures, and the $(b-y)$ temperatures. Also shown are the L16 values. The shaded region indicates a quadratic sum of typical $(1 \sigma) \mathrm{Fe}$ I and Fe II uncertainties $( \pm 0.14 \mathrm{dex})$.

shifting them to higher or lower values. This is another possible source of difference between our study and L16 that could directly affect $\delta \mathrm{Fe}$, and thus it requires investigation.
As a first step we directly compared our Fe I and Fe II $\log g f$ values with those used by $\mathrm{L}_{16}{ }^{10}$. For Fe I we found the average difference for our 40 lines to be $\Delta \log g f=-0.02 \operatorname{dex}(\sigma=0.09$; in the sense L16 - this study). Since final abundances are taken as an average over the abundances inferred from each line, and considering other uncertainties, this difference is insignificant ${ }^{11}$. For the Fe II lines the average difference is slightly larger, being $\Delta \log g f=+0.05$ dex. However, for Fe II we only used two or three lines, so in the cases where only two lines were available, even one significantly deviant $\log g f$ value would be expected to alter the derived abundances tangibly - and thus alter $\delta \mathrm{Fe}$ by offsetting Fe II. To check the sensitivity of our results to this difference we re-derived $\mathrm{Fe}$ abundances for our entire stellar sample using the L16 $\log g f$ values for Fe II. The Fe II lines $(\Delta \log g f)$ we used were: $6149.23 \AA$ (+0.04 dex), $6247.56 \AA$ (+0.02 dex), and $6369.46 \AA$ (+0.10 dex). All else was kept constant.

The results of this analysis are presented in Table 1 . The slightly increased Fe II oscillator strengths led to an average decrease of $[\mathrm{Fe} \mathrm{II} / \mathrm{H}]$ of $0.05 \mathrm{dex}$ in both the RGB and AGB samples. As Fe I is unchanged, this leads to correspondingly minor changes in $\delta \mathrm{Fe}$. For the RGB sample $\delta \mathrm{Fe}$ becomes marginally significant $(+0.09 \mathrm{dex})$, albeit in the opposite sense to the original problem in the AGB sample. In the AGB stars $\delta \mathrm{Fe}$ remains insignificant, with $\delta \mathrm{Fe}$ changing from -0.04 to $+0.01(\sigma=$ 0.08).

Thus the adopted $\log g f$ values appear to contribute to the uncertainty in $\delta \mathrm{Fe}$ only to a small degree. Additionally, the lower abundance derived from Fe II using the L16 oscillator strengths most likely explains part of the $\sim 0.1$ dex lower average $[\mathrm{Fe}$ II $/ \mathrm{H}]$ value found by L16.

\subsubsection{NLTE effects}

Our final $\delta \mathrm{Fe}$ results appear to show a weak trend toward higher values at higher $T_{\text {eff }}$ in the AGB sample (Fig. 12). This could be due to a real overionisation of Fe I, but the overionisation effect

${ }^{10}$ L16 used the Kurucz/Castelli line list for all species except for Fe II, for which they used values from Meléndez \& Barbuy (2009).

${ }^{11}$ We also compared our Fe I $\log g f$ values to those used by the GaiaESO Survey (Ruffoni et al. 2014). Here also the average difference is minor, with $\Delta \log g f=0.02 \operatorname{dex}(\sigma=0.09)$. There are however only five lines in common (out of 40). 
would have to be stronger in stars in this particular temperature range $\left(T_{\text {eff }}>4800 \mathrm{~K}\right)$.

The magnitude of NLTE effects in atmospheres of cool stars has been recently studied by Lind et al. (2012) and Mashonkina et al. (2016). Figure 2 of Lind et al. (2012) shows that, at the metallicity of NGC 6752, the NLTE corrections for $\mathrm{Fe} I$ are expected to be small for AGB and RGB stars.

As a check we computed the expected NLTE corrections for a range of $\mathrm{Fe} I$ lines at some characteristic parameters of our AGB and RGB stars using both the Lind et al. (2012) and Mashonkina et al. (2016) web-based interpolation routines ${ }^{12}$. We found that corrections were consistent between the two compilations. The corrections were also almost constant, varying by just \pm 0.01 dex, so they are basically offsets. The constancy across the AGB temperature range implies that the marginal $\delta \mathrm{Fe}$ trend in Fig. 12 is not explained by current NLTE theory. The magnitude of the corrections are however slightly different across each set of stars, with $\Delta_{\mathrm{NLTE}}$ averaging +0.05 dex for the RGB stars and +0.09 dex for the AGB stars. The slightly higher value for AGB stars is expected due to their higher temperatures $^{13}$.

The NLTE offsets increase the average $\delta \mathrm{Fe}$ to +0.09 in the RGB sample, and to +0.05 (from -0.04) in the AGB sample. Considering other uncertainties, these offsets are small. It is important to recognise that the NLTE corrections themselves also have uncertainties. Lind et al. (2011) showed that model atmosphere choice alone can alter the predicted NLTE corrections by up to $\sim 0.1$ dex (their Fig. 8, for Na I). This is comparable to the magnitude of the predicted offsets we have reported here. To be consistent with L16, and considering the small effect on the results, we did not apply the NLTE offsets to our final Fe I results. This also avoids adding in the extra uncertainty of the corrections themselves.

\subsubsection{Model atmospheres}

The choice of model atmosphere has an effect on abundance determinations. This is due to the fact that different physical stratifications are predicted by different stellar atmosphere codes for the same set of stellar parameters. It is the differences in adopted physical descriptions in each set of theoretical models that gives rise to the different stratifications. For example, some use "pure MLT" to describe convection, whilst some use modified MLT formalisms. Overshoot (see e.g. Castelli et al. 1997) and the adopted treatment of opacity are other model variables.

We ran some tests to gauge the effect of using different model grids on the derived Fe I and Fe II abundances. For the tests we used four different grids: a MARCS grid ${ }^{14}$ (Gustafsson et al. 2008), and three different Kurucz/Castelli grids ${ }^{15}$ (their NOVER, OVER, and AODFNEW grids; Kurucz 1993; Castelli \& Kurucz 2004). The four grids differ in terms of overshoot (or lack of), treatment of convection, and treatment of opacity, for example. The parameters and EWs of one RGB star (star 12) and one AGB star (star 97) were used.

12 The INSPECT interface: http://www.inspect-stars.com (Lind et al. 2011), and the interface by Mashonkina et al. (2016): http://spectrum.inasan.ru/nLTE

13 The lower gravity of the AGB stars compared to RGB stars at the same temperature reduces the difference marginally.

${ }^{14}$ Downloaded from http://marcs . astro.uu.se

15 Downloaded from http://kurucz . harvard. edu/grids.html
We found differences of 0.04 to 0.12 dex in the derived Fe I and Fe II abundances, with Fe I consistently at the upper end. This is consistent with the uncertainty due to adopted model atmospheres reported by Lind et al. (2011) in relation to NLTE corrections. This $\sim 0.1$ dex uncertainty is especially important when considering species that are very temperature sensitive, here Fe I, since the temperature stratification changes significantly. This test also indicates that uncertainties of this order must be allowed for when comparing between independent studies, even if they are based on the same data, since the model grid choice affects the results. Variations in tools/pipelines that make use of the model grids must also add to these uncertainties.

Two other possible sources of uncertainty from model atmospheres are (i) the choice of plane parallel or spherical (but still 1D) models; and (ii) the choice of model stellar mass. Traditionally, $1 M_{\odot}$ stellar atmospheres are used for GC stars, since there is negligible effect in changing the mass by small amounts. However, due to the particularly low masses of AGB stars $\left(\sim 0.6 M_{\odot}\right)$, models with mass of $0.5 M_{\odot}$ may be more appropriate. Due to their low envelope mass, the atmospheres of these stars are expected to be more extended than those of RGB stars, and thus spherical effects may be important. To check these two factors we made a test using the MARCS $0.5 M_{\odot}$ spherical models, for which only a small grid exists (Gustafsson et al. 2008). We compared the Fe I and Fe II abundances derived using MARCS models with mass of $1 M_{\odot}$ with those derived using $0.5 M_{\odot}$ models, for a star with characteristic AGB parameters. We found that the differences were negligible, being of the order $0.01 \mathrm{dex}$. This indicates that mass and sphericity are not important in the case of these AGB stars.

Finally we note that the discussion above has only involved 1D model atmospheres. Three dimensional model atmospheres are now becoming available and have been shown to have significantly different stratifications as compared to 1D models (e.g. Magic et al. 2013). Thus the use of 3D model atmospheres would be expected to introduce further differences in abundance determinations.

\subsubsection{Distance}

The cluster distance is a fundamental parameter that has a direct impact on the gravity scale through the derivation of the stellar bolometric magnitudes. All of the studies (C13, L16 and this study) used the Harris (1996) catalogue value of ( $m-$ $M)_{V}=13.13$. A literature search showed that this is at the lower end of the values published, which range from 13.13 to 13.38 (just in the studies we consulted, the range may be greater; Renzini et al. 1996; Gratton et al. 2003; Yong et al. 2005). Taking the maximum of these values systematically decreases gravity by $0.1 \mathrm{dex}$. This uncertainty in $\log g$ translates to a systematic shift of the microturbulent velocity scale by an insignificant amount (+0.03 dex, based on the Gratton et al. 1996 relation). Nevertheless, we tested the effect of these small systematic shifts using the parameters of AGB star 97 and RGB star 12. As expected, Fe I was unaffected and Fe II was reduced by $\sim 0.05$ dex. This reduced $\delta \mathrm{Fe}$ in the AGB star, from -0.14 to -0.10 dex, and increased it in the RGB star since Fe I was already greater than Fe II. Again these are small changes but they do add to the many other small uncertainties. 


\subsubsection{Effect of AGB stellar mass on gravity}

Another uncertainty affecting gravity determination is the adopted stellar mass for the AGB stars. The median HB stellar mass was estimated at $0.61 M_{\odot}$ for NGC 6752 by Gratton et al. (2010). This was adopted by L16 and the current study. According to theory, lower masses are possible. For example, Dorman et al. (1993) find a minimum envelope mass for AGB ascension of $0.035 M_{\odot}$, at the metallicity of NGC 6752. Adding their core mass of $0.48 M_{\odot}$ suggests that the minimum mass for an AGB star should be $0.52 M_{\odot}$. This difference of $\sim-0.1 M_{\odot}$ would systematically reduce the gravity of the AGB stars by 0.07 dex. Importantly this would only affect AGB stars, leaving the RGB gravities unchanged. However the AGB masses may also be higher. Assuming a normal distribution of AGB masses around $0.61 M_{\odot}$ we thus (roughly) estimate a $1 \sigma$ error of $\sim \pm 0.05$ dex on the surface gravity due to the uncertainty in total stellar mass. As discussed above this can cause small changes in abundance results, particularly in the Fe abundance derived from Fe II.

\subsubsection{Summary of uncertainties}

Here we have only explored some of the uncertainties inherent in spectroscopic abundance determination. From this investigation it is clear that, apart from the large uncertainty in $T_{\text {eff }}$ given by some colour- $T_{\text {eff }}$ relations, and the uncertainties in measuring EWs, there are many other sources of uncertainty that lead to additional abundance differences of the order $0.01 \rightarrow 0.10$ dex. All the uncertainties must combine, probably in a non-linear way, to create "noise" and systematic shifts in the results, increasing the uncertainty of our final abundances. It is also clear that each source of uncertainty (including $T_{\text {eff }}$ ) affects Fe I and Fe II to different degrees. It would thus be expected that each ion of each element would also be affected to different degrees. For a broader view of uncertainties in spectroscopic abundance determination we refer the interested reader to the detailed empirical study of Hinkel et al. (2016).

\section{Sodium from $\mathrm{C} 13$ data using new $T_{\text {eff }}$ scale}

Of primary interest to the argument of $\mathrm{C} 13$ (and to a lesser extent L16) is the distribution of sodium amongst the AGB stars. It is possible that the new IRFM/( $V-K)$ temperature scale (Fig. 9) could remove the good agreement between $[\mathrm{Na} / \mathrm{H}]$ between studies (Fig. 2), given that the offset is $\sim 100 \mathrm{~K}$.

$\mathrm{NaI}$ is predicted to suffer NLTE effects in giant stars such as those studied here. C13 and L16 both used the Gratton et al. (1999) corrections to LTE abundances. For the current study we have chosen to use the more recent NLTE corrections calculated by Lind et al. (2011). As noted by Lind et al. (2011) the Gratton et al. (1999) corrections differ from most tabulations in the literature, especially at low temperatures and gravities. The Na I lines we have used are: 5682.6 $\AA$, 5688.2 $\AA, 6154.2 \AA$, and $6160.7 \AA$. The last two of these lines were generally not detectable in the AGB stars, but a total of three or four lines were usually detectable in the RGB stars.

We computed NLTE corrections for all available Na lines for all stars using the INSPECT web interface ${ }^{16}$ (Lind et al. 2011). The corrections were not large, with an average of -0.08 dex in the AGB sample, and -0.10 dex for the RGB sample. In both

\footnotetext{
16 http://wWW. inspect-stars.com
}

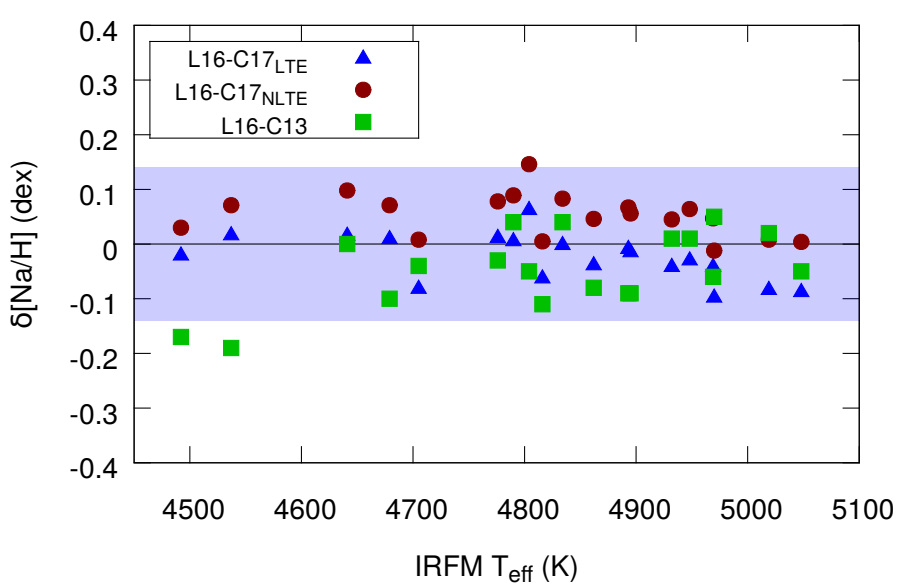

Fig. 13. Comparison between our new AGB Na abundances (labelled C17), the $\mathrm{C} 13$ abundances, and those of L16. All abundance sets are corrected for NLTE effects except C17 $7_{\mathrm{LTE}}$, which is included to highlight the magnitude of the corrections. The shaded region denotes typical uncertainties of $0.1 \mathrm{dex}$, added in quadrature ( $\sigma=0.14 \mathrm{dex}$ in total). The two coolest stars are discussed in the text.

sets of stars this was essentially an offset, with the standard deviation of the corrections being just $\sigma=0.01 \mathrm{dex}$ (AGB) and 0.02 dex (RGB). All corrections and final abundances are listed in Table A.1.

In the RGB sample the average standard deviation of the line-to-line abundance scatter, $\sigma_{\text {ave }}$, was reduced from $0.08 \mathrm{dex}$ (LTE) to $0.06 \operatorname{dex}$ (NLTE). In the AGB stars $\sigma_{\text {ave }}$ reduced from 0.04 dex to 0.03 dex. Although these are small changes this is reassuring as it is what is expected if the corrections are of the correct sign and magnitude. We note that since the AGB stars usually only have two lines measured, the abundances in the AGB stars shouldn't be taken as more accurate than those of the RGB stars. Indeed, we find the average line-to-line scatter increases with the number of lines measured in the AGB stars, with $\sigma_{\text {ave }}=0.10$ dex in the stars with three detectable lines (the RGB stars generally have three or four lines measurable). This is an important point - it is common practice to report standard deviations of very small samples of lines as uncertainties in abundances. This can lead to overconfidence in results.

In Fig. 13 we compare our new $[\mathrm{Na} / \mathrm{H}]$ values with those of L16 and C13. Somewhat surprisingly, it can be seen that practically all stars have essentially the same abundances in all three studies, within the uncertainties. The only exceptions are the two coolest stars: the current study and L16 find substantially higher $\mathrm{Na}$ than $\mathrm{C} 13$ for these stars. This appears to be a minor error in $\mathrm{C} 13$, although it has no effect on the conclusions of that study.

The average difference in Na abundance between L16 and the current study, in the sense L16-this study, is +0.05 dex $(\sigma=0.04)$. Between L16 and C13 it is $-0.05 \operatorname{dex}(\sigma=0.07)$, excluding the 2 coolest stars. It is interesting that the increase in temperature of $\sim 100 \mathrm{~K}$ (above the scale of L16) has no significant effect on the $\mathrm{Na}$ abundances. This does concur with the observation that a roughly $40 \mathrm{~K}$ temperature difference between C13 and L16 also had no significant effect (Fig. 2). Adding to this that different tools, model atmospheres, different stellar mass assumptions, and different spectra were used between the studies strongly suggests that the $\mathrm{Na}$ abundances are very robust, at least within the current analysis framework.

Our RGB sample has an average abundance of $[\mathrm{Na} / \mathrm{H}]=$ -1.38 dex, with a standard deviation of $\sigma=0.27$ dex. In 
contrast, the AGB sample has an average abundance of $[\mathrm{Na} / \mathrm{H}]=$ -1.71 dex, with a standard deviation of $\sigma=0.13$ dex. While the spread in the RGB sample is much larger than the uncertainties, the scatter in the AGB sample is low, and similar to the 0.10 dex reported by $\mathrm{C} 13$. Indeed, $\mathrm{C} 13$ noted that this level of scatter was similar to their uncertainties and therefore consistent with a single abundance of $\mathrm{Na}$. We explore this topic further in the Discussion.

\section{Discussion and conclusion}

We have investigated the differences between the NGC 6752 AGB star abundance studies of $\mathrm{C} 13$ and L16. In this paper we have focused on $\mathrm{Fe}$ and $\mathrm{Na}$, since $\mathrm{L} 16$ reported a very strong apparent overionisation of $\mathrm{Fe}$ I, and argued that all neutral species, including $\mathrm{Na}$ I, should be scaled by $\mathrm{Fe}$ I, thus altering the distribution of $[\mathrm{Na} / \mathrm{Fe}]$ as compared to $\mathrm{C} 13$ (Fig. 3).

By dividing all neutral species by Fe derived from Fe I, L16 essentially made the assumptions that:

1. overionisation affects all neutral species;

2. they are affected by exactly the same magnitude of overionisation.

While (1) is possible, (2) is highly unlikely, since the magnitudes of NLTE effects are known to vary between species, and indeed between lines of the same species. There are also variations with $T_{\text {eff }}$, gravity, and abundance of the element (see e.g. Lind et al. 2011, 2012 for the cases of $\mathrm{Na}$ and $\mathrm{Fe}$ ).

The scaling to Fe I for neutral species in GC AGB stars was originally suggested by Ivans et al. (2001). They however proposed it as one of three options to reconcile Fe I and Fe II in the AGB stars of the GC M5. These options were arrived at after an extensive investigation into the many possible causes of the $\mathrm{Fe}$ discrepancy. The rationale behind the Fe I scaling option was due to the observation that: "Whenever Fe appears to be overionized ... Si, Ti, and $\mathrm{V}$ are excessively ionized by essentially the same amount..." (Ivans et al. 2001). This was then generalised to include all neutral species. As Ivans et al. (2001) state, this was an extrapolation - it was not based on any theoretical calculations, since they weren't available at that time. These calculations are now available, in particular for Fe I and $\mathrm{Na}$ I. In the case of Fe I the predicted NLTE corrections (Sect. 5.2.2) are much smaller than the depression of Fe I reported by L16 (Fig. 4). This has created a "tension" between theory and observations (see also Lapenna et al. 2014).

During our comparison tests we immediately reproduced a strong apparent depression of Fe I, similar to L16. Significantly, this was achieved with different input data and computational tools. However further investigation showed that this is primarily driven by the adopted stellar temperatures. By deriving more reliable temperatures specifically for our stellar sample via the IRFM method we found that the putative Fe I overionisation became insignificant. By comparing temperatures derived with colour-Teff relations to our IRFM temperatures it was found that $(V-K)$ relations are the most reliable (they have much smaller uncertainties than $(B-V)$ relations), and we suggest that they be used for future (early) AGB studies. Previous AGB studies that have reported strong apparent Fe I overionisation will need to be checked.

Interestingly, one of the possibilities canvassed by Ivans et al. (2001) to remove the Fe discrepancy was indeed to "arbitrarily increase the values of $T_{\text {eff }}$ above the Alonso et al. scale by $60 \mathrm{~K}$ on the RGB and $120 \mathrm{~K}$ on the AGB". By investigating other options for temperature scales they found that there were scales that were systematically hotter (see their Sect. 3.3 for details). They concluded that this avenue to resolve the Fe discrepancy "remains an option".

Related to this is the study of 47 Tuc by Lapenna et al. (2014). Noting that their finding of a strong depression of Fe I in the AGB stars was at odds with theoretical predictions, they made a detailed investigation into the uncertainties affecting $\delta \mathrm{Fe}$. They excluded $T_{\text {eff }}$ as a significant source of uncertainty because the excitation balance was "well satisfied" in their sample. Our result - that spectroscopically determined temperatures tend to lie close to the initial estimates (usually photometric) may be the reason that Lapenna et al. (2014) could not reconcile the 47 Tuc AGB Fe I and Fe II abundances. An indication that this may be the case is given by one of the tests performed by Lapenna et al. (2014). They found that $\delta \mathrm{Fe}$ became negligible if parameters from a higher mass star $\left(1.2 M_{\odot}\right)$ were used. The $T_{\text {eff }}$ increase above the nominal value was $\sim+100 \mathrm{~K}$. The fact that this was a positive $T_{\text {eff }}$ offset that reduced $\delta \mathrm{Fe}$ matches with our findings (Fig. 7). The magnitude of this $T_{\text {eff }}$ offset is similar to the offsets we have found here (Fig. 9), so we would expect a greater increase in Fe I, of the order $\sim 0.2$ dex as compared to $\sim 0.1$ dex found by Lapenna et al. (2014). However the much higher metallicity of 47 Tuc may reduce the effect.

L16 also discussed their results in terms of $[\mathrm{X} / \mathrm{H}]$, noting that using $\mathrm{Fe}$ in the denominator may have skewed the results (they report that it did not). However the different $T_{\text {eff }}$ scale presented here ${ }^{17}$ means that all elements need reanalysis, since the effects are uncertain and most likely vary from element to element, as our investigation of $\mathrm{Fe}$ and $\mathrm{Na}$ has shown. As molecular band formation is highly dependent on temperature, abundances for elements based on molecules ( $\mathrm{C}, \mathrm{N}$, and indirectly, $\mathrm{O}$, in L16) are likely to be altered. We are unsure how the results of the L16 study will be affected. The effects may or may not alter the conclusions on the topic of AGB subpopulations, but it clearly requires investigation.

After the reconciliation of Fe I and Fe II, we checked the effect of the new temperature scale on derived sodium abundances and found that it had no significant effect. Interestingly the $[\mathrm{Na} / \mathrm{H}]$ abundances across the three studies $(\mathrm{C} 13, \mathrm{~L} 16$, and the current study) all agree, within the uncertainties. It is also remarkable that such large differences in temperatures, gravity, input data, and tools, have such little effect on the Na abundances. This suggests they are very robust - at least in the current paradigm of 1D stellar atmosphere abundance determinations and NLTE modelling. We speculate that either (i) significant changes in the (currently small) predicted NLTE corrections; or (ii) 3D atmosphere effects, would be the most likely factors that could alter the $\mathrm{Na}$ abundances. Since $\mathrm{Fe}$ is constant in NGC 6752 then $[\mathrm{Na} / \mathrm{Fe}]$ must also be consistent between all studies, aside from possible systematic offsets in Fe.

We found that the $\mathrm{Na}$ abundance spread in the AGB sample is slightly larger than that found by $\mathrm{C} 13(\sigma=0.13$ dex versus 0.10 dex). This compares with a spread of $\sigma=0.27$ dex in the RGB sample. As shown in C13 the AGB distribution is centered over the RGB SP1 distribution. However we are cautious to assign significance to the slight increase in the AGB dispersion, especially given the exploration of the many sources of uncertainty in Sect. 5.2. In particular we note the uncertainties in NLTE corrections that could amount to $0.1 \mathrm{dex}$, but which are generally ignored in abundance studies. We also recall that the $\mathrm{Na}$

\footnotetext{
${ }^{17}$ Gravity should be only slightly affected, with changes most likely smaller than the L16 uncertainty of $\sim 0.1$ dex (Sect. 3).
} 
abundances in the AGB stars are usually based on just two lines, so the line-to-line scatter uncertainty is not well constrained. Indeed, we found that the line-to-line scatter increases in the stars with more $\mathrm{Na}$ lines measured, to a an average value of $0.10 \mathrm{dex}$ in the AGB stars with three detectable lines ${ }^{18}$. As this is just one source of uncertainty, it should be taken as a minimum for the total abundance uncertainty. That said, our empirical result in Fig. 13, which shows that the Na abundances appear very robust between the three studies (all within \pm 0.05 dex, on average), may suggest that the spread is significant ${ }^{19}$. If it is, this could be considered a small signal of the tail of the second population distribution being present on the AGB, similar to that found for M4 (see Discussion in MacLean et al. 2016). However, given the small magnitude of the signal, it is advisable to attempt to identify the subpopulations in multi-dimensional chemical space instead, as noted by others (e.g. L16; MacLean et al. 2016). Thus we leave the further discussion of AGB subpopulations to our next paper in the series, where we will investigate other elements given the improved AGB temperatures. What appears certain is that the Na spread in the AGB stars is very restricted compared to the RGB stars.

We conclude by noting that care must be taken in deriving AGB star temperatures, and, more generally, that uncertainty reporting in abundance analysis papers should be more robust there are many sources of uncertainty that can significantly alter the results. The relevant example here is the standard procedure of testing the abundance results' sensitivity to temperature. Both C13 and L16 used an estimated uncertainty of $\sim \Delta T_{\text {eff }} \pm 30 \mathrm{~K}$. This uncertainty is small compared to the $T_{\text {eff }}$ differences of $\sim 60-100 \mathrm{~K}$ found here. If more realistic uncertainties were included, the error bars on Fe I would have been large $\mathrm{e}^{20}-$ and the results consistent with theory and other abundance studies.

Acknowledgements. The spectroscopic observations were carried out with the Very Large Telescope under ESO programme 089.D-0038 (PI Campbell). Part of this work was supported by the DAAD (PPP project 57219117) with funds from the German Federal Ministry of Education and Research. D.Y. acknowledges funding from the Australian Research Council (FT140100554). B.T.M acknowledges the financial support of the Australian Postgraduate Award scholarship. We thank Yazan Momany and Frank Grundahl for providing their photometric datasets, and Yue Wang and Francesca Primas for helpful discussions. This work made extensive use of the SIMBAD, Vizier, 2MASS, and NASA ADS databases. It also made use of the open-source software packages MOOG (Chris Sneden; http://www.as.utexas.edu/ chris/moog.htmland) and $q 2$ (Ivan Ramirez; https://github.com/astroChasqui/q2). We thank the anonymous referee for their constructive comments, which improved the paper.

\section{References}

Alonso, A., Arribas, S., \& Martínez-Roger, C. 1999, A\&AS, 140, 261

Baraffe, I., Chabrier, G., Allard, F., \& Hauschildt, P. H. 1997, A\&A, 327, 1054

Campbell, S. W., Lattanzio, J. C., \& Elliott, L. M. 2006, Mem. Soc. Astron. Ital., 77,864

Campbell, S. W., Yong, D., Wylie-de Boer, E. C., et al. 2010, Mem. Soc. Astron. Ital., 81, 1004

Campbell, S. W., D’Orazi, V., Yong, D., et al. 2013, Nature, 498, 198

18 This compares with the LTE (NLTE) average scatter of 0.08 (0.06) dex in the RGB stars, which mostly have three or four lines measured.

${ }_{19}$ Although it may instead reflect that usually the same two Na lines are used in every AGB study, and, as we have shown, that these particular lines are insensitive to many factors.

${ }^{20}$ See final paragraph of Sect. 4.2.1.
Campbell, S. W., Constantino, T. N., D’Orazi, V., et al. 2016, Astron. Nachr., 337,788

Carretta, E., Bragaglia, A., Gratton, R. G., Lucatello, S., \& Momany, Y. 2007, A\&A, 464, 927

Carretta, E., Bragaglia, A., Gratton, R., D’Orazi, V., \& Lucatello, S. 2009, A\&A, 508,695

Carretta, E., Bragaglia, A., Gratton, R. G., et al. 2010, A\&A, 516, A55

Carretta, E., Bragaglia, A., Gratton, R. G., Lucatello, S., \& D’Orazi, V. 2012, ApJ, 750, L14

Casagrande, L., Ramírez, I., Meléndez, J., Bessell, M., \& Asplund, M. 2010, A\&A, 512, A54

Cassisi, S., Salaris, M., Pietrinferni, A., Vink, J. S., \& Monelli, M. 2014, A\&A, 571, A81

Castellani, V., \& Renzini, A. 1968, Ap\&SS, 2, 310,

Castelli, F., \& Kurucz, R. L. 2004, Proc. IAU Symp. 210, poster A20 [arXiv:astro-ph/0405087]

Castelli, F., Gratton, R. G., \& Kurucz, R. L. 1997, A\&A, 318, 841

Chantereau, W., Charbonnel, C., \& Meynet, G. 2016, A\&A, 592, A111

Constantino, T., Campbell, S. W., Christensen-Dalsgaard, J., Lattanzio, J. C., \& Stello, D. 2015, MNRAS, 452, 123

Constantino, T., Campbell, S. W., Lattanzio, J. C., \& van Duijneveldt, A. 2016 , MNRAS, 456, 3866

D’Antona, F., Caloi, V., Montalbán, J., Ventura, P., \& Gratton, R. 2002, A\&A, 395,69

D’Antona, F., Bellazzini, M., Caloi, V., et al. 2005, ApJ, 631, 868

de Silva, G. M., Gibson, B. K., Lattanzio, J., \& Asplund, M. 2009, A\&A, 500, L25

Dorman, B., Rood, R. T., \& O'Connell, R. W. 1993, ApJ, 419, 596

Fitzpatrick, E. L., \& Massa, D. 2007, ApJ, 663, 320

García-Hernández, D. A., Mészáros, S., Monelli, M., et al. 2015, ApJ, 815, L4

González Hernández, J. I., \& Bonifacio, P. 2009, A\&A, 497, 497

Gratton, R. 1988, Rome Obs. Preprint Ser., 29

Gratton, R. G., \& Sneden, C. 1988, A\&A, 204, 193

Gratton, R. G., Carretta, E., \& Castelli, F. 1996, A\&A, 314, 191

Gratton, R. G., Carretta, E., Eriksson, K., \& Gustafsson, B. 1999, A\&A, 350, 955

Gratton, R. G., Bragaglia, A., Carretta, E., et al. 2003, A\&A, 408, 529

Gratton, R. G., Bragaglia, A., Carretta, E., et al. 2005, A\&A, 440, 901

Gratton, R. G., Carretta, E., Bragaglia, A., Lucatello, S., \& D’Orazi, V. 2010, A\&A, 517, A81

Gratton, R. G., Carretta, E., \& Bragaglia, A. 2012, Astron. Astrophys. Rev., 20, 50

Gratton, R. G., Lucatello, S., Sollima, A., et al. 2015, A\&A, 573, A92

Gray, D. F. 2005, The Observation and Analysis of Stellar Photospheres, 3rd edn. (Cambridge University Press)

Grevesse, N., \& Sauval, A. J. 1998, Space Sci. Rev., 85, 161

Grundahl, F., Catelan, M., Landsman, W. B., Stetson, P. B., \& Andersen, M. I. 1999, ApJ, 524, 242

Gruyters, P., Casagrande, L., Milone, A. P., et al. 2017, A\&A, 603, A37

Gustafsson, B., Edvardsson, B., Eriksson, K., et al. 2008, A\&A, 486, 951

Harris, W. E. 1996, AJ, 112, 1487

Hinkel, N. R., Young, P. A., Pagano, M. D., et al. 2016, ApJS, 226, 4

Iben, Jr., I. 1971, PASP, 83, 697

Ivans, I. I., Kraft, R. P., Sneden, C., et al. 2001, AJ, 122, 1438

Johnson, C. I., McDonald, I., Pilachowski, C. A., et al. 2015, AJ, 149, 71

Karakas, A. I., Marino, A. F., \& Nataf, D. M. 2014, ApJ, 784, 32

Kurucz, R. L. 1993, SYNTHE spectrum synthesis programs and line data (Cambridge: Smithsonian Astrophysical Observatory)

Lapenna, E., Mucciarelli, A., Lanzoni, B., et al. 2014, ApJ, 797, 124

Lapenna, E., Mucciarelli, A., Ferraro, F. R., et al. 2015, ApJ, 813, 97

Lapenna, E., Lardo, C., Mucciarelli, A., et al. 2016, ApJ, 826, L1

Lardo, C., Salaris, M., Savino, A., et al. 2017, MNRAS, 466, 3507

Lind, K., Asplund, M., Barklem, P. S., \& Belyaev, A. K. 2011, A\&A, 528, A103

Lind, K., Bergemann, M., \& Asplund, M. 2012, MNRAS, 427, 50

MacLean, B. T., Campbell, S. W., De Silva, G. M., et al. 2016, MNRAS, 460, L69

MacLean, B. T., De Silva, G. M., \& Lattanzio, J. 2015, MNRAS, 446, 3556

Magic, Z., Collet, R., Hayek, W., \& Asplund, M. 2013, A\&A, 560, A8

Mallia, E. A. 1978, A\&A, 70, 115

Marino, A. F., Villanova, S., Milone, A. P., et al. 2011, ApJ, 730, L16

Mashonkina, L. I., Sitnova, T. N., \& Pakhomov, Y. V. 2016, Astron. Lett., 42, 606

Meléndez, J., \& Barbuy, B. 2009, A\&A, 497, 611

Milone, A. P. 2015, MNRAS, 446, 1672

Momany, Y., Piotto, G., Recio-Blanco, A., et al. 2002, ApJ, 576, L65

Mucciarelli, A. 2011, A\&A, 528, A44

Mucciarelli, A., Lapenna, E., Massari, D., Ferraro, F. R., \& Lanzoni, B. 2015, ApJ, 801, 69 
Norris, J. 1974, ApJ, 194, 109

Norris, J., Cottrell, P. L., Freeman, K. C., \& Da Costa, G. S. 1981, ApJ, 244, 205

Pasquini, L., Alonso, J., Avila, G., et al. 2003, in Instrument Design and Performance for Optical/Infrared Ground-based Telescopes, eds. M. Iye, \& A. F. M. Moorwood, Proc. SPIE, 4841, 1682

Piotto, G., Bedin, L. R., Anderson, J., et al. 2007, ApJ, 661, L53

Ramírez, I., \& Meléndez, J. 2005, ApJ, 626, 465

Renzini, A., Bragaglia, A., Ferraro, F. R., et al. 1996, ApJ, 465, L23

Ruffoni, M. P., Den Hartog, E. A., Lawler, J. E., et al. 2014, MNRAS, 441, 3127

Salaris, M., Cassisi, S., \& Pietrinferni, A. 2016, A\&A, 590, A64

Schlegel, D. J., Finkbeiner, D. P., \& Davis, M. 1998, ApJ, 500, 525

Schwarzschild, M. 1970, Quant. J. Roy. Astron. Soc., 11, 12
Skrutskie, M. F., Cutri, R. M., Stiening, R., et al. 2006, AJ, 131, 1163 Sneden, C. A. 1973, Ph.D. Thesis, The university of Texas at Austin Sneden, C., Ivans, I. I., \& Kraft, R. P. 2000, Mem. Soc. Astron. Ital., 71, 657 Stetson, P. B. 2000, PASP, 112, 925

Sweigart, A. V. 1997, ApJ, 474, L23

Valcarce, A. A. R., Catelan, M., Alonso-García, J., Contreras Ramos, R., \& Alves, S. 2016, A\&A, 589, A126

Wang, Y., Primas, F., Charbonnel, C., et al. 2016, A\&A, 592, A66

Yong, D., Grundahl, F., Nissen, P. E., Jensen, H. R., \& Lambert, D. L. 2005, A\&A, 438, 875

Yong, D., Grundahl, F., \& Norris, J. E. 2015, MNRAS, 446, 3319

Zinn, R. 1974, ApJ, 193, 593 


\section{Appendix A}

Table A.1. Final stellar parameters and abundance results.

\begin{tabular}{|c|c|c|c|c|c|c|c|c|c|c|c|c|c|}
\hline ID & Type & $\begin{array}{l}T_{\text {eff }} \\
(\mathrm{K})\end{array}$ & $\begin{array}{l}\log g \\
(\operatorname{dex})\end{array}$ & $\begin{array}{c}\mathrm{Xi} \\
\left(\mathrm{km} \mathrm{s}^{-1}\right)\end{array}$ & $\begin{array}{c}\text { Fe I } \\
(\operatorname{dex})\end{array}$ & $\begin{array}{c}\sigma \\
(\operatorname{dex})\end{array}$ & $\begin{array}{l}\text { Fe II } \\
(\operatorname{dex})\end{array}$ & $\begin{array}{c}\sigma \\
(\mathrm{dex})\end{array}$ & $\begin{array}{c}\mathrm{Na} \mathrm{I}_{\mathrm{LTE}} \\
(\mathrm{dex})\end{array}$ & $\begin{array}{c}\sigma \\
(\operatorname{dex})\end{array}$ & $\begin{array}{c}\text { Corr }_{\mathrm{Na} I} \\
(\mathrm{dex})\end{array}$ & $\begin{array}{c}\mathrm{Na} \mathrm{I}_{\mathrm{NLTE}} \\
(\mathrm{dex})\end{array}$ & $\begin{array}{c}\sigma \\
(\mathrm{dex})\end{array}$ \\
\hline 22 & AGB & 4641 & 1.31 & 1.80 & 6.01 & 0.11 & 5.92 & 0.06 & 4.83 & 0.06 & -0.08 & 4.75 & 0.05 \\
\hline 25 & AGB & 4492 & 1.09 & 1.87 & 6.07 & 0.10 & 6.03 & 0.11 & 4.56 & 0.10 & -0.05 & 4.51 & 0.11 \\
\hline 31 & AGB & 4537 & 1.20 & 1.83 & 6.08 & 0.11 & 5.99 & 0.09 & 4.52 & 0.04 & -0.05 & 4.47 & 0.03 \\
\hline 44 & AGB & 4679 & 1.43 & 1.76 & 6.03 & 0.12 & 6.03 & 0.13 & 4.52 & 0.01 & -0.06 & 4.46 & 0.00 \\
\hline 52 & AGB & 4862 & 1.68 & 1.68 & 5.96 & 0.10 & 6.01 & 0.16 & 4.70 & 0.04 & -0.08 & 4.61 & 0.03 \\
\hline 53 & AGB & 4790 & 1.57 & 1.72 & 5.99 & 0.08 & 6.01 & 0.07 & 4.76 & 0.03 & -0.08 & 4.68 & 0.02 \\
\hline 59 & AGB & 4804 & 1.61 & 1.70 & 5.92 & 0.10 & 5.94 & 0.08 & 4.71 & 0.03 & -0.08 & 4.62 & 0.02 \\
\hline 60 & AGB & 4776 & 1.57 & 1.72 & 6.12 & 0.12 & 6.05 & 0.12 & 4.54 & 0.00 & -0.07 & 4.47 & 0.01 \\
\hline 61 & AGB & 4834 & 1.63 & 1.70 & 6.04 & 0.10 & 6.01 & 0.09 & 4.73 & 0.04 & -0.08 & 4.65 & 0.02 \\
\hline 65 & AGB & 4705 & 1.43 & 1.76 & 6.00 & 0.10 & 6.02 & 0.05 & 4.95 & 0.05 & -0.09 & 4.86 & 0.05 \\
\hline 75 & AGB & 4816 & 1.67 & 1.68 & 5.91 & 0.07 & 6.05 & 0.11 & 4.50 & 0.01 & -0.07 & 4.44 & 0.00 \\
\hline 76 & AGB & 4970 & 1.76 & 1.65 & 5.92 & 0.11 & 5.97 & 0.09 & 4.89 & 0.14 & -0.08 & 4.80 & 0.15 \\
\hline 78 & AGB & 4948 & 1.76 & 1.65 & 5.93 & 0.12 & 5.97 & 0.10 & 4.86 & 0.04 & -0.09 & 4.77 & 0.02 \\
\hline 80 & AGB & 4893 & 1.75 & 1.66 & 5.95 & 0.09 & 6.07 & 0.07 & 4.59 & 0.04 & -0.07 & 4.51 & 0.03 \\
\hline 83 & AGB & 4932 & 1.76 & 1.65 & 5.96 & 0.09 & 6.03 & 0.15 & 4.71 & 0.01 & -0.09 & 4.63 & 0.00 \\
\hline 94 & AGB & 4969 & 1.83 & 1.63 & 5.90 & 0.07 & 6.08 & 0.10 & 4.73 & 0.07 & -0.09 & 4.64 & 0.06 \\
\hline 97 & AGB & 5048 & 1.89 & 1.61 & 5.92 & 0.10 & 6.06 & 0.08 & 4.78 & 0.02 & -0.09 & 4.69 & 0.03 \\
\hline 104 & AGB & 4895 & 1.79 & 1.64 & 5.94 & 0.10 & 6.07 & 0.19 & 4.48 & 0.00 & -0.07 & 4.41 & 0.00 \\
\hline 201620 & AGB & 5019 & 1.83 & 1.63 & 6.01 & 0.11 & 6.01 & 0.04 & 4.84 & 0.01 & -0.09 & 4.75 & 0.02 \\
\hline 12 & RGB & 4348 & 1.10 & 1.87 & 6.09 & 0.12 & 5.92 & 0.09 & 5.10 & 0.05 & -0.08 & 5.01 & 0.07 \\
\hline 23 & RGB & 4404 & 1.19 & 1.84 & 6.09 & 0.10 & 6.12 & 0.02 & 5.12 & 0.08 & -0.09 & 5.04 & 0.04 \\
\hline 27 & RGB & 4453 & 1.30 & 1.80 & 6.08 & 0.13 & 6.13 & 0.04 & 4.77 & 0.18 & -0.06 & 4.70 & 0.18 \\
\hline 29 & RGB & 4362 & 1.13 & 1.86 & 6.07 & 0.11 & 6.07 & 0.17 & 4.67 & 0.04 & -0.06 & 4.61 & 0.05 \\
\hline 30 & RGB & 4362 & 1.10 & 1.87 & 6.09 & 0.12 & 5.93 & 0.11 & 5.10 & 0.18 & -0.09 & 5.01 & 0.13 \\
\hline 35 & RGB & 4490 & 1.37 & 1.78 & 6.09 & 0.12 & 6.05 & 0.05 & 5.39 & 0.13 & -0.11 & 5.28 & 0.08 \\
\hline 43 & RGB & 4469 & 1.37 & 1.78 & 6.04 & 0.09 & 5.99 & 0.07 & 5.45 & 0.11 & -0.12 & 5.33 & 0.04 \\
\hline 50 & RGB & 4436 & 1.27 & 1.81 & 6.11 & 0.12 & 6.08 & 0.16 & 4.95 & 0.06 & -0.09 & 4.86 & 0.03 \\
\hline 54 & RGB & 4571 & 1.51 & 1.73 & 6.18 & 0.11 & 6.08 & 0.15 & 4.94 & 0.03 & -0.09 & 4.85 & 0.03 \\
\hline 64 & RGB & 4467 & 1.36 & 1.78 & 6.07 & 0.09 & 5.98 & 0.11 & 5.39 & 0.15 & -0.12 & 5.27 & 0.08 \\
\hline 91 & RGB & 4641 & 1.75 & 1.66 & 5.99 & 0.12 & 6.01 & 0.12 & 5.10 & 0.06 & -0.09 & 5.01 & 0.06 \\
\hline 92 & RGB & 4672 & 1.73 & 1.66 & 6.12 & 0.11 & 6.02 & 0.13 & 4.72 & 0.11 & -0.08 & 4.64 & 0.12 \\
\hline 107 & RGB & 4681 & 1.83 & 1.63 & 6.07 & 0.12 & 5.99 & 0.10 & 5.03 & 0.08 & -0.09 & 4.94 & 0.09 \\
\hline 129 & RGB & 4738 & 1.94 & 1.60 & 6.03 & 0.12 & 6.08 & 0.20 & 5.04 & 0.05 & -0.10 & 4.94 & 0.06 \\
\hline 155 & RGB & 4752 & 2.00 & 1.58 & 6.02 & 0.13 & 6.01 & 0.09 & 4.62 & 0.01 & -0.08 & 4.54 & 0.02 \\
\hline 161 & RGB & 4852 & 2.07 & 1.55 & 6.14 & 0.09 & 6.05 & 0.10 & 5.23 & 0.06 & -0.12 & 5.11 & 0.04 \\
\hline 170 & RGB & 4792 & 2.07 & 1.55 & 6.07 & 0.14 & 6.05 & 0.08 & 5.40 & 0.08 & -0.12 & 5.27 & 0.02 \\
\hline 186 & RGB & 4832 & 2.13 & 1.54 & 6.02 & 0.15 & 5.99 & 0.13 & 4.63 & 0.06 & -0.08 & 4.54 & 0.04 \\
\hline 193 & RGB & 4854 & 2.14 & 1.53 & 6.06 & 0.14 & 6.11 & 0.08 & 4.58 & 0.00 & -0.08 & 4.50 & 0.01 \\
\hline 262 & RGB & 4873 & 2.25 & 1.50 & 6.03 & 0.09 & 5.92 & 0.04 & 5.17 & 0.04 & -0.12 & 5.06 & 0.01 \\
\hline 276 & RGB & 4864 & 2.25 & 1.50 & 5.99 & 0.09 & 6.02 & 0.08 & 5.20 & 0.10 & -0.12 & 5.08 & 0.06 \\
\hline 200619 & RGB & 4731 & 1.91 & 1.61 & 6.02 & 0.07 & 6.06 & 0.09 & 5.41 & 0.01 & -0.08 & 5.33 & 0.01 \\
\hline
\end{tabular}

Notes. Abundances are presented as $\log \epsilon=\log \left(\mathrm{N}_{\mathrm{X}} / \mathrm{N}_{\mathrm{H}}\right)+12$, where $\mathrm{X}$ represents each species. The $\sigma$ values are based on line-to-line abundance scatter only. The NLTE corrections to the LTE sodium abundances are given in the third last column. 